PART 9

VARIABLE X-RAY SOURCES 


\title{
THE BINARY X-RAY STARS - THE OBSERVATIONAL PICTURE
}

\author{
HERBERT GURSKY and ETHAN SCHREIER \\ Center for Astrophysics, Harvard College Observatory/Smithsonian Astrophysical Observatory, \\ Cambridge, Mass., U.S.A.
}

\section{Introduction}

When X-ray sources in the galaxy were discovered in 1962 (Giacconi et al., 1962) it was only possible to speculate on their nature, which centered on supernova and cosmic-ray phenomena since these were the only very energetic events known. In particular, the discovery of an X-ray source associated with the Crab Nebula led to the idea that the emission might be the thermal radiation from the surface of a hot, neutron star. However, it was soon demonstrated (Bowyer et al., 1964) that a neutron star could not be responsible for the bulk of the X-radiation from the Crab, and it was not possible to exclude highly pathological conditions in otherwise ordinary stellar systems as being responsible for the $\mathrm{X}$-ray sources (cf. Hayakawa and Matsuoka, 1964).

Since that time, there have been fundamental advances in astronomy and in the physics of compact stars. The quasars and the pulsars have been discovered and the generation of enormous quantities of non-thermal power on a galactic scale is clearly demonstrated. Partially stimulated by the discovery of X-ray sources and of pulsars, substantial progress has been made in the physics of neutron stars and black holes the physical conditions within these objects and in their vicinity, the manner in which they are formed and their likely appearance in an astronomical setting. The rôle of Soviet astrophysicists, particularly Professor Shklovsky and Acad. Zeldovich, is noteworthy in this respect. In a general way, both emphasized the significant rôle that neutron stars and black holes might have in the production of the observed X-rays. Specifically, Shklovsky (1967) showed that the optical and X-ray emission from Sco X-1 could reasonably be accounted for as originating in a complex plasma surrounding a neutron star powered by an accretion from a nearby companion. And Zeldovich and Shakura (1969) demonstrated quantitatively that the plasma in the vicinity of a neutron star would be at a temperature of $10^{7}-10^{8} \mathrm{~K}$, rather than $10^{12} \mathrm{~K}$ obtained by naive calculations. In an apparently unrelated activity, astronomers became concerned with how the evolution of stars is affected by their being in close binary systems where mass transfer might be important as seemed to be the case in certain stellar systems such as the old novae. These studies are now believed to be an important consideration in the evolution of neutron stars and black holes.

Until the last few years, little of this work could be tied directly to observational X-ray astronomy. However, beginning in late 1970, with the launching of NASA's $\mathrm{X}$-ray satellite, 'Uhuru', a wealth of new observational material has been accumulated. 
The quantity of data has produced a qualitative change in our understanding of the field. The change has been so great that it is almost as if a new field of astronomy has been created. In particular, it can be argued that the galactic $\mathrm{X}$-ray sources are ordinary binary stellar systems in which one member is a compact star. Furthermore, it can be argued that the compact star is more likely to be a black hole or a neutron star rather than a white dwarf. The reasons for this view are that,

(1) Many of the sources are positively confirmed as being in binary systems.

(2) The short time variability of the X-ray emission requires a compact emission region.

(3) Mass accretion onto a neutron star or black hole from its nearby companion should be an extremely efficient means of generating $X$-rays.

The field has come full circle in the sense that the supernova remnants, which provided the early incentive for associating X-ray emission with compact stars, may be the least interesting of the sources. Except for sorting them out, they will not be discussed further. The prominent galactic X-ray sources are listed in Table I. As in the other branches of non-optical astronomy, the sources with identified optical counterparts have an unusual status since for these it is possible to determine fundamental characteristics such as distance, mass, age, etc. However, because of restrictions of both X-ray and optical observations, only nine optical identifications have been made.

Seven of the 9 identified sources are binary systems; however, as will be discussed further, the binary character is a particularly easy one to distinguish observationally.

TABLE I

Outstanding galactic $\mathrm{X}$-ray sources

\begin{tabular}{|c|c|c|c|c|}
\hline $3 \mathrm{U}$ designation & Name & $\begin{array}{l}\text { Binary } \\
\text { period }\end{array}$ & $\begin{array}{l}\text { X-ray } \\
\text { characteristics }\end{array}$ & $\begin{array}{l}\text { Optical } \\
\text { characteristics }\end{array}$ \\
\hline $0115-37$ & SMC X-1 & 3.9 days & $\begin{array}{l}\text { X-ray eclipse } \\
\text { extended lows }\end{array}$ & 13 mag. BOIb \\
\hline $0900-40$ & Vela XR-1 & 8.9 days & $\begin{array}{l}\text { X-ray eclipse } \\
\text { slow (h) flares }\end{array}$ & 6 mag. B0.5Ib \\
\hline $1118-60$ & Cen X-3 & 2.1 days & $\begin{array}{l}\text { X-ray eclipse } 4.8 \mathrm{~s} \text { pulse } \\
\text { period extended lows }\end{array}$ & 13 mag. BOIb \\
\hline $1617-15$ & Sco X-1 & - & Slow (min-h) flares & 12-13 mag. non-stellar \\
\hline $1653+35$ & Her X-1 & 1.7 days & $\begin{array}{l}\text { X-ray eclipse } 1.2 \mathrm{~s} \text { pulse } \\
\text { period } 35 \text { day high/low } \\
\text { cycle }\end{array}$ & $13-15$ mag. - late $A$ \\
\hline $1700-37$ & & 3.4 days & $\begin{array}{l}\text { X-ray eclipse irregular } \\
\text { variability to } 0.1 \mathrm{~s}\end{array}$ & $6 \mathrm{mag} .07 \mathrm{f}$ \\
\hline $1956+35$ & Cyg X-1 & 5.6 days & $\begin{array}{l}\text { Irregular variability } \\
\text { to } 1 \mathrm{~min}\end{array}$ & 9 mag. O8.7Ib \\
\hline $2030+40$ & Cyg X-3 & $4.8 \mathrm{~h}$ & $\begin{array}{l}\sim \text { sinusoidal } 4.8 \mathrm{~h} \\
\text { variation }\end{array}$ & IR source \\
\hline
\end{tabular}

Seat of intense radio outbursts

$2142+38 \quad$ Cyg X-2 $\quad$ _ Irregular variability to min. 14 mag. G 
Nevertheless, it is possible that the preponderance of binaries in Table I reflects the preponderance of binaries among all of the galactic X-ray sources. In fact, it can be argued that all the $\mathrm{X}$-ray sources are binaries.

One group of the identified sources is associated with a veryp articular kind of stars a late $\mathbf{O}$ or early $\mathbf{B}$ supergiant, a star which is very massive and very luminous. The relative rarity of this kind of star probably means that the star itself is a necessary ingredient in the presence of the X-ray emission in these systems. Other X-ray sources are clearly not associated with 0-B stars, but rather with much later spectral type stars - stars which are much more like the Sun in temperature, luminosity and mass.

Thus, two very different kinds of stellar systems contain the X-ray sources. The $\mathrm{X}$-ray data themselves do not reveal any particular homogeneity in their characteristics. The temporal structure of the radiation is found to be highly variable on every time scale from milliseconds to years, and the variability is both random and periodic. One of these X-ray sources, Cyg X-1, is most credibly interpreted as a black hole. Its $\mathrm{X}$-ray emission is very highly variable on a time scale down to $1 \mathrm{~ms}$, and its mass is significantly greater than can be accommodated by other compact stars, namely, white dwarfs or neutron stars. Two other X-ray sources, Cen X-3 and Her X-1, are likely to be rapidly rotating neutron stars. Their X-ray emission is almost precisely periodic with periods of 4.8 and $1.2 \mathrm{~s}$, respectively.

This paper presents the current observational evidence on these objects both from an astronomical and astrophysical point of view. We discuss first the distributional properties of the sources, where they appear in the galaxy, and certain average characteristics. In this way, certain properties of the X-ray sources can be deduced which are not apparent in the study of single objects. We then go on to describe the properties of individual X-ray sources.

\section{General Characteristics of the Galactic X-Ray Sources}

X-ray astronomy has encompassed both the study of the properties of individual sources and the study of the average characteristics of the sources. The latter, while unsatisfactory in many respects, is the only manner in which certain properties of the sources can be determined. It frequently takes the form of studying the degree of correlation of the spatial distribution of the X-ray emission with other, better known distributions of cosmic radiation. It is the information that can be obtained from this kind of study that is reviewed here.

The general feature of the distribution of the galactic sources, namely, a strong concentration of sources at low galactic longitude, was established during the earliest rocket experiments. The first observations that approached the angular resolution of current instruments and showed that there were a large number of discrete sources strung along the Milky Way were those of Fisher et al. (1966) and of Friedman et al. (1967). The latter study also included an attempt to enumerate the number of sources based on guessing the location of sources within specific spiral arms. Gursky et al. (1967) attempted to demonstrate a specific correlation with spiral arms based on the 
similarity of the longitude distribution of the $X$-ray sources, and $21 \mathrm{~cm}$ radio emission, $\mathrm{H}$ II regions and O-B associations. The latter are the accepted spiral arm indicators. Ryter (1970) and Setti and Woltjer (1970) made an important contribution to this kind of study by pointing out the importance of a galactic 'ridge', (an excess flux of radiation along the Milky Way which could be attributed to unresolved sources) in estimating the average distance to the resolved sources and the space-density of sources.

With the availability of Uhuru data, these distributional studies could be made more quantitative than they had in the past as was shown by Salpeter (1973) and Gursky (1973). Salpeter analyzed the latitude and longitude distribution of the sources in the central region of the galaxy and the consequences for a ridge. His principal conclusion was that the bright sources at low galactic longitude were not population I. Gursky analyzed the same data and argued that the sources outside the central region still showed a correlation with the spiral arms. The general conclusions from these studies was as follows:

(1) The luminosity of the sources is within the range $10^{35-38} \mathrm{erg} \mathrm{s}^{-1}$.

(2) The number of sources in the Galaxy in this luminosity range is not large, perhaps no more than 100 .

(3) The X-ray luminosity is concentrated in the range 2-10 keV. Possibly most of the X-ray sources are surrounded by an absorbing envelope of the order of $10^{22}$ atoms $\mathrm{cm}^{-2}$ of hydrogen. In particular, the various low energy $(<1 \mathrm{keV})$ rocket surveys (cf. Seward et al., 1972) fail to reveal a significant number of sources not seen at higher energy.

(4) The sources in the outer regions of the Galaxy are found in the spiral arms and are thus of extreme population $I$.

(5) The bright sources in the central region are not of extreme population I, but rather seem to be distributed as are the ordinary stars.

\subsection{RESULTS FROM THE 3U CATALOG}

The newly prepared $3 U$ catalog (Giacconi et al., 1974) can be used to extend these conclusions. This catalog lists 161 discrete sources ranging in intensity from the most intense Sco X-1 at $3 \times 10^{-7} \mathrm{erg} \mathrm{cm}^{-2} \mathrm{~s}^{-1}$ to the faintest at about $10^{4}$ times weaker. The distribution of all these sources is shown in Figure 1 which is a plot in galactic coordinates of the source positions. The sources are divided into intensity classes based on the photon counting rate observed by the Uhuru instruments in the 2-6 keV energy range (a count rate of $1 \mathrm{ct} \mathrm{s}^{-1}$ is approximately $1.7 \times 10^{-11} \mathrm{erg} \mathrm{cm}^{-2} \mathrm{~s}^{-1}$ ).

The striking feature about the distribution is the concentration of strong sources along the Milky Way, especially at low galactic longitude $(l)$. The sources at high galactic latitude $(b)$ are much weaker. In fact, many of them can be identified with specific extragalactic objects, and it can be argued, based on their distribution that most of them must be extragalactic. The only exceptions to this are identified objects, i.e., Her X-1 and Sco X-1, which are both galactic sources. It is the strong sources which concern us here. They make up the great majority of the identified galactic 


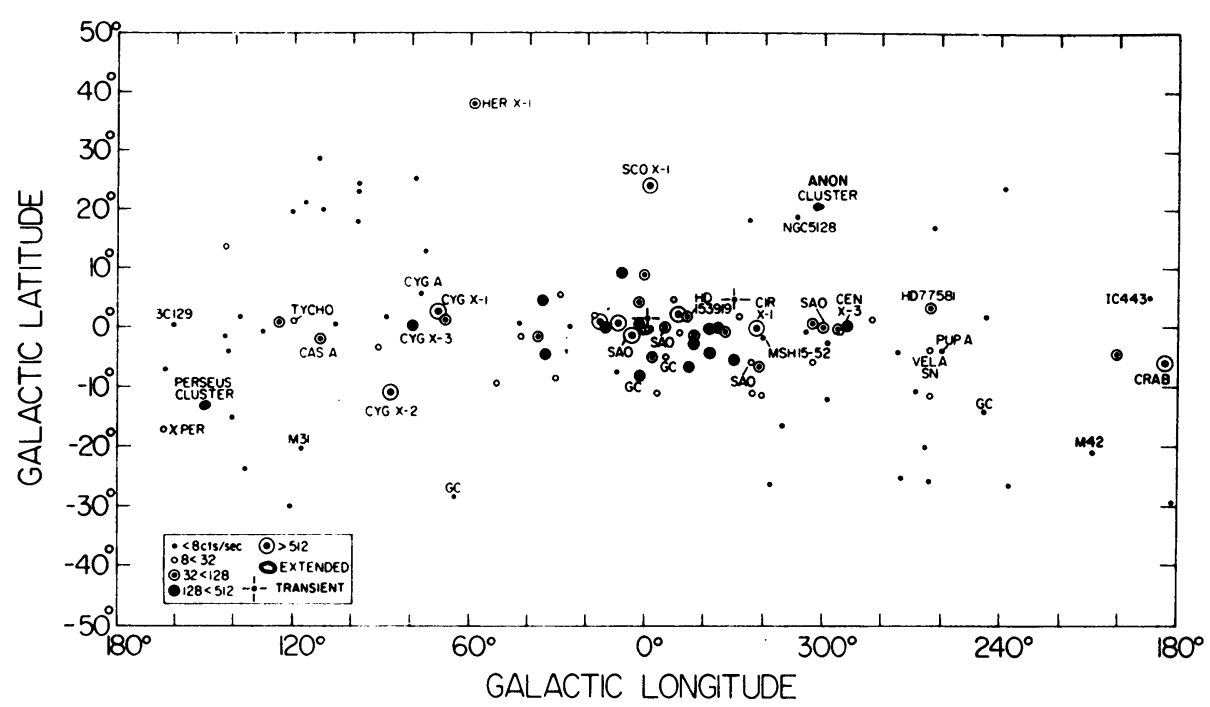

Fig. 1. The distribution of X-ray sources from the $3 \mathrm{U}$ Catalog galactic coordinates. Only low latitude sources are plotted.

sources and contain those individual objects which have come to exemplify the field as a whole. There is also a class of weak $\left(<8 \mathrm{ct} \mathrm{s}^{-1}\right)$ sources associated with the Galaxy. These can be seen only at large angular separation from the galactic center and along the Milky Way where the density of strong sources is not great; in particular, in the interval $80-160^{\circ} \mathrm{l}$. Within the band $\pm 5^{\circ}$ of latitude, there are $163 \mathrm{U}$ sources $<8 \mathrm{ct} \mathrm{s}^{-1}$ whereas only 5 are expected based on the all-sky density of such weak sources.

The galactic X-ray sources are listed in the Appendix. The first portion of the list includes those sources with $\mathrm{I}>8 \mathrm{ct} \mathrm{s}^{-1}$, which are not identified with wellknown galactic objects such as supernovae. These sources are listed in order of galactic longitude beginning at $320^{\circ}$. It is likely that all of these are galactic. As will be discussed below, the region $320-40^{\circ}$ delineates the central region of the Galaxy. We list separately the single sources seen in the Magellanic Clouds and, for completeness, the sources $<8 \mathrm{ct} \mathrm{s}^{-1}$ which are within $5^{\circ}$ of the galactic plane. It is likely that many of these are extragalactic. Also listed separately are the transient sources, those objects which were seen for one period of time but not otherwise. In addition to galactic coordinates, the Appendix lists the $3 \mathrm{U}$ name, which is the right ascension (hours and minutes) and declination (degrees) of the source, the intensity in Uhuru counts and the variability, being the ratio between the maximum and minimum daily average intensity with which the source was observed. A comments column lists other significant features regarding the X-ray emission, including optical candidates.

\subsection{EVIDENCE FOR EXTREME POPULATION I SOURCES}

The X-ray sources associated with massive B0 supergiants, such as Cygnus $\mathrm{X}-1$ and 
3U 0900-40, are clearly of extreme population I. The significance of this is their youth - it can be no more than about $10^{7} \mathrm{yr}$ since they have condensed out of the interstellar medium. These objects are presumably examples of the class of sources associated with spiral arms and exhibiting a very small dispersion in galactic latitude.

The distributional evidence for an association with spiral arms is actually very weak. The Cygnus region is frequently cited as an example of such an association. As can be seen from the Appendix, only 3 sources $>8 \mathrm{ct} \mathrm{s}^{-1}$ lie in the range $70-90^{\circ}$ longitude which delineate that arm; however, of those sources, Cyg X-3 is now known to be about $10 \mathrm{kpc}$ distant, far outside the limits of this arm, and Cyg X-2 will be cited below as belonging to a different population. Thus, the 'association' reduces to the single object Cyg X-1, which is hardly significant.

The Centaurus Arm, the region $280-300^{\circ}$ longitude, seems to be a better delineated feature since it contains $7 \mathrm{X}$-ray sources and is followed $\left(304-320^{\circ}\right)$ by a significant region devoid of sources.

The X-ray sources that may be of extreme population I are listed in Table II.

TABLE II

Extreme population I X-ray sources

3U designation $\quad l, b \quad Z(\mathrm{pc}) \quad I\left(\mathrm{ct} \mathrm{s}^{-1}\right) \quad \begin{aligned} & \text { Distance } \\ & (\mathrm{kpc})\end{aligned} \quad L\left(\mathrm{erg} \mathrm{s}^{-1}\right)$

Positively identified

$\begin{array}{llrccr}0900-40 & (263,4) & 90 & 100 & 1.3 & 3.2 \times 10^{36} \\ 1118-60(\text { Cen X-3) } & (292,0) & 60 & 350 & 10 & 6 \times 10^{37} \\ 1700-37 & (348,2) & 65 & 102 & 1.7 & 6 \times 10^{37} \\ 1956+35(\text { Cyg X-1) } & (71,3) & 130 & 1175 & 2.5 & 1.4 \times 10^{37} \\ 0115-37(\text { SMC X-1) } & - & & 20 & 60 & 1.4 \times 10^{38}\end{array}$

Candidate objects

$\begin{array}{rrrl}0352+30 & (163,-17) & 20 & \text { Optical object? } \\ 1145-61 & (296,0) & 72 & \text { B0, 9.2 m; B2, 8.6 m } \\ 1556-60 & (324,-5) & 17 & \text { B9, 8.9 m } \\ 1624-49 & (335,0) & 50 & \text { B8, 9 m, NGC 6134 } \\ 1727-33 & (354,0) & 65 & \text { B5, 6.7 m; B8, 9.4 m }\end{array}$

The average latitude of the identified sources is $\sim 2^{\circ}$, and the average distance from the galactic plane is about $90 \mathrm{pc}$ based on the distance estimate to the companion stars. This is as expected for an extreme population I. The spread in X-ray luminosity is from $3 \times 10^{35}-10^{38} \mathrm{erg} \mathrm{s}^{-1}$.

Further evidence for extreme population I objects came from the statistical association of X-ray sources with bright B stars found by Gursky (1972) using the selected sources from the $2 U$ catalog. Such an association was first suggested by Liller (1972c). When this is done with the $3 \mathrm{U}$ catalog, out of 41 unidentified sources with positional uncertainty less than $0.02 \mathrm{deg}^{2}$, ten are found to be associated with stars listed in the 
SAO catalog whereas the number of accidental associations is expected to be fewer than three. Of the ten, five are associated with B spectral type stars and, surprisingly for two of these, 3U $1727-33$ and $3 U 1145-61$, two B stars are within the X-ray area positional uncertainty. These associations are listed as possible candidates in Table II. These plus other possible stellar associations are listed in the Appendix in the comments on individual sources.

The probability of having two B stars within a single X-ray error box in two instances is extremely small. This may be a reflection of the tendency of B stars to be found in clusters; thus, the B stars are not randomly distributed but rather tend to group together. In fact, one X-ray source $3 \mathrm{U} 1624-49$ may be within the star cluster NGC 6134.

\subsection{EVIDENCE FOR OLD POPULATION SOURCES}

Several X-ray sources are known not to be associated with massive B0 stars. These sources are listed in Table III. In particular, the optical companion of Her X-1 is late $A$, that of Cyg X-2 is either of F or G spectral type, and no stellar companion can be detected for Sco X-1.* Thus, these objects could be common, low mass stars,

TABLE III

Old population X-ray sources

\begin{tabular}{llcccc} 
3U designation & $(l, b)$ & $Z(\mathrm{pc})$ & $I\left(\mathrm{ct} \mathrm{s}^{-1}\right)$ & $\begin{array}{l}\text { Distance } \\
(\mathrm{Kpc})\end{array}$ & $\begin{array}{l}\text { Luminosity } \\
\left(\mathrm{erg} \mathrm{s}^{-1}\right)\end{array}$ \\
\hline Positively identified & & & & & \\
$1617-15($ Sco X-1) & $(359,+24)$ & $80-800$ & 17000 & $0.2-2$ & $10^{36}-10^{38}$ \\
$1653+35($ Her X-1) & $(58,+38)$ & 3700 & 100 & 6 & $7 \times 10^{36}$ \\
$2030+40($ Cyg X-3) & $(80,+1)$ & 120 & 194 & 10 & $4 \times 10^{37}$ \\
$2142+38($ Cyg X-2) & $(87,-11)$ & 120 & 540 & 0.6 & $4 \times 10^{35}$ \\
Candidate objects & & & & & Optical object? \\
$1746-37$ & $(354,-5)$ & & 31 & \multicolumn{2}{l}{ Glob. Cluster, NGC 6441 } \\
$1820-30$ & $(3,-8)$ & & 250 & \multicolumn{2}{c}{ Glob. Cluster, NGC 6624 }
\end{tabular}

much like the Sun, either of population I or population II. It is noteworthy that three of the sources lie at the largest latitudes $-38^{\circ}, 11^{\circ}, 24^{\circ}$, respectively - of any of the galactic X-ray sources. Cyg X-3 is the fourth object with a low mass companion, based on the assumption that it is a binary system with a 0.2 day period. A massive star can not be a member of such a short period binary system. The range in luminosity of these sources is $4 \times 10^{35}-4 \times 10^{37} \mathrm{erg} \mathrm{s}^{-1}$.

On piece of evidence favoring a population II halo origin is that two X-ray sources, $3 \mathrm{U} 1746-37$ and $3 \mathrm{U} 1820-30$, are coincident with the globular clusters NGC 6441 and NGC 6624, which is statistically a very unlikely event.

* Sco X-1 is seen as $+13 \mathrm{~m}$ at minimum intensity. In order for there to be present an equal intensity of light from a BO supergiant (which could hardly go undetected) the distance to Sco X-1 would need to be $\sim 50 \mathrm{kpc}$, which is well outside our Galaxy. 


\subsection{VERY LOW LUMINOSITY SOURCES}

The lowest luminosity of an identified source is $\sim 4 \times 10^{35} \mathrm{erg} \mathrm{s}^{-1}$; however, there is evidence for sources of lower luminosity. One source, $3 \mathrm{U} 0352+30$ is coincident with the star X Persei. This star is cited as possibly being a runaway from the Perseus OB2 association of O-B stars (Blaauw, 1961). On this basis, its distance is about $300 \mathrm{pc}$ and the luminosity of the X-ray source about $4 \times 10^{33} \mathrm{erg} \mathrm{s}^{-1}$.

Another piece of evidence for low luminosity sources is the excess of weak $\left(<8 \mathrm{ct} \mathrm{s}^{-1}\right)$ sources found along the Milky Way in the interval 80-160 longitude. If their distance is the order of $5 \mathrm{kpc}$, their luminosity is about $4 \times 10^{35}$, which is at the lowest end of luminosity of the identified sources. Thus, they could be merely more distant examples of known sources.

\subsection{ARE ALL THE X-RAY SOURCES IN CLOSE BINARY SYSTEMS?}

We can estimate the probability of seeing a binary system in eclipse in X-rays by making assumptions on the mass of the system and the radius of the larger star. If the X-ray source is assumed to be a point, the probability of eclipse is simply,

$$
P(\text { eclipse })=r / a,
$$

where $r=$ radius of the star, $a=$ separation of star and X-ray source, and from Kepler's laws, for circular orbits,

$$
a=(T / 2 \pi)^{2 / 3}\left[G\left(m_{1}+m_{2}\right)\right]^{1 / 3},
$$

where $T=$ binary period, and $m_{1}, m_{2}=$ mass of the binary components. Then

$$
P(\text { eclipse })=0.19 r / T^{2 / 3} m^{1 / 3},
$$

where $r$ is now in units of solar radii, $T$ is in units of days, and $m$ is in units of solar masses.

Three X-ray sources of the extreme population I category $(0900-40$, Cen X-3, and $1700-37)$ are seen to eclipse with periods between 2.1 and 8.9 days. Assuming the total mass to be $20 \mathrm{~m}_{\odot}$, the probability of eclipse of these objects varies between 16 and $43 \%$, if the radius of the B star is taken to be $10 r_{\odot}$ and between 32 and $85 \%$ if the radius is taken to be $20 r_{\odot}$. Having seen these three in eclipse, we would expect there to be between 5 and 10 systems of this kind in the Galaxy with the same strength.

In the case of the two eclipsing sources of the older population (Her X-1 and Cyg X-3) we assumed the total mass to be $2 m_{\odot}$ and take the radius to be either 1 or $2 r_{\odot}$. Again, we expect between 5 and 10 systems of this kind making a total of 10-20. In fact, 25 galactic X-ray sources are seen with $I>100 \mathrm{ct} \mathrm{s}^{-1}$. Considering the fact that longer period binaries are less likely to eclipse and more difficult to discover, the data are consistent with all the X-ray sources being in binary systems of the kind seen in eclipse. However, these numbers are also consistent with $\sim 1 / 3$ to $1 / 2$ of the $\mathrm{X}$-ray sources not being in binary systems at all. 


\subsection{SPACE DENSITY AND LUMINOSITY OF THE X-RAY SOURCES}

As discussed above, the identified $X$-ray sources lie at distances ranging from $500 \mathrm{pc}$ to $10 \mathrm{kpc}$ and have luminosities from $10^{35}-10^{38} \mathrm{erg} \mathrm{s}^{-1}$. This information by itself provides a qualitative answer to the question of the space density and luminosity of the X-ray sources. A more general answer can be found by looking at the distribution of all the sources. To aid in discussing these sources we have plotted their distribution in latitude-intensity space in Figure 2. The sources are divided between two regions: the central region between $320^{\circ}<l<40^{\circ}$ and the outer region $40^{\circ}<l<320^{\circ}$. Most of the sources in the central region may belong to the central bulge of our Galaxy. In Table IV, we list the correspondence between intensity in Uhuru counts and lumi-

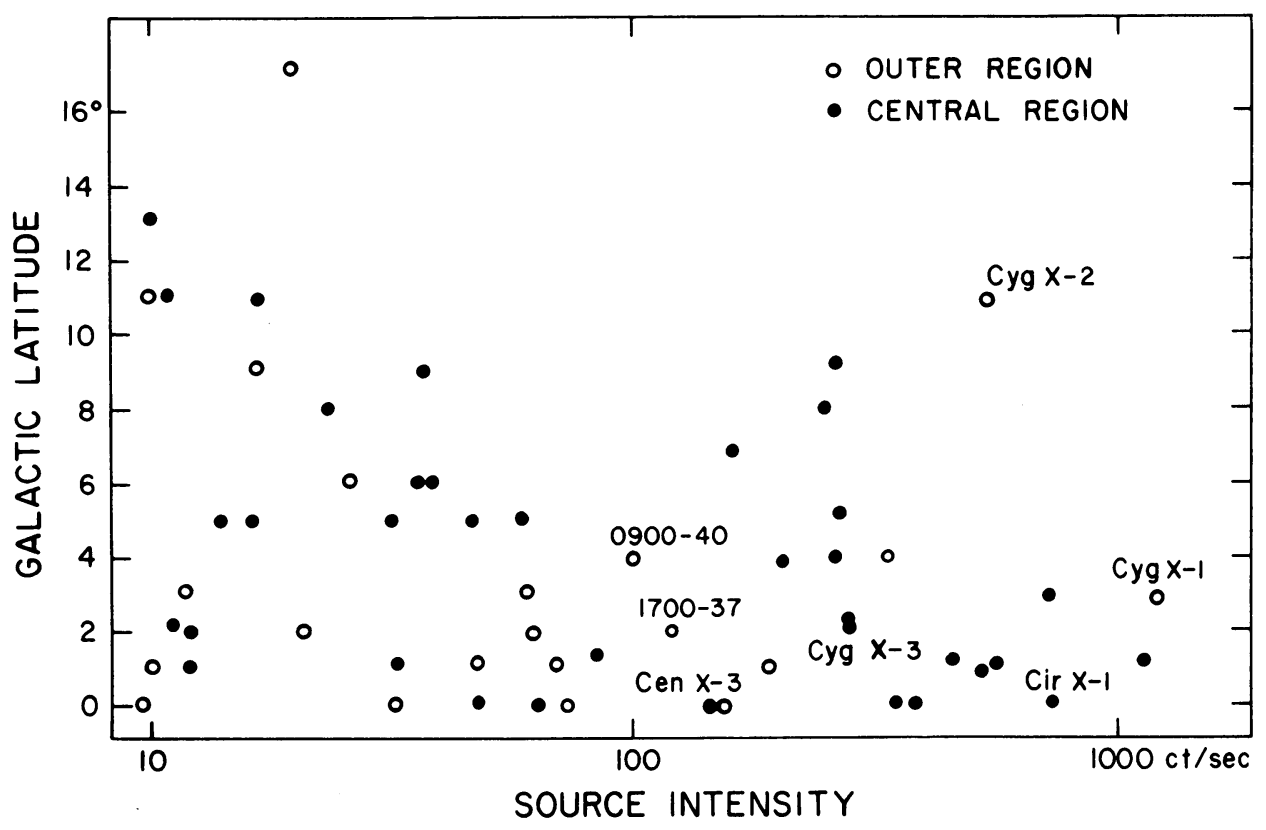

Fig. 2. The intensity-latitude distribution of X-ray sources from the $3 \mathrm{U}$ Catalog. Open circles are those sources appearing in the central region of the Galaxy. The closed circles are sources in the outer region of the Galaxy. Sco X-1 and Her X-1 have not been plotted.

TABLE IV

Correspondence between luminosity $\left(\mathrm{erg} \mathrm{s}^{-1}\right)$, distance and Uhuru counting rate

\begin{tabular}{rllll}
$\begin{array}{l}\text { Uhuru count rate } \\
\text { (ct s }\end{array}$ & \multicolumn{4}{l}{ Distance $(\mathrm{kpc})$} \\
\cline { 2 - 5 } & 1 & 2 & 5 & 10 \\
\hline 10 & $2 \times 10^{34}$ & $8 \times 10^{34}$ & $5 \times 10^{35}$ & $2 \times 10^{36}$ \\
30 & $6 \times 10^{34}$ & $2.5 \times 10^{35}$ & $1.5 \times 10^{36}$ & $6 \times 10^{36}$ \\
100 & $2 \times 10^{35}$ & $8 \times 10^{35}$ & $5 \times 10^{36}$ & $2 \times 10^{37}$ \\
300 & $6 \times 10^{35}$ & $2.5 \times 10^{36}$ & $1.5 \times 10^{37}$ & $6 \times 10^{37}$ \\
1000 & $2 \times 10^{36}$ & $8 \times 10^{36}$ & $5 \times 10^{37}$ & $2 \times 10^{38}$
\end{tabular}


nosity. To a good approximation, this luminosity is the total power between 2-6 keV radiated by the source and for many of the sources is close to the total radiated power.

In the outer region, it is likely that all sources $>8 \mathrm{ct} \mathrm{s}^{-1}$ have been detected by Uhuru, with the possible exception of those lying in the Centaurus region where source confusion could still be a problem. Since there is no great increase of sources at low intensity, it is likely that these sources are distributed throughout the outer portion of the galaxy out to distances of the order of $10 \mathrm{kpc}$. What we mean by this is simply that 5 sources with $I>100 \mathrm{ct} \mathrm{s}^{-1}$ and 19 sources with $I>10 \mathrm{st} \mathrm{s}^{-1}$ are seen. If the sources were distributed in a disc, their number would increase as $1 / I$, which is clearly not the case. Also, the identified sources range in distance from $600 \mathrm{pc}$ for Cyg X-2 to $\sim 10 \mathrm{kpc}$ for both Cen X-3 and Cyg X-3. Thus, the fainter sources in this region are likely to be a mix between more distant and lower luminosity sources.

The central region is more complex since it is likely that we fail to see a significant number of the faint sources due to confusion. It is commonly believed that the bright sources in this region ( $I$ greater than several hundred cts $\mathrm{s}^{-1}$ ) are $\sim 10 \mathrm{kpc}$ distant and are similar to those found in the Magellanic Clouds (cf. Gursky, 1973; Margon and Ostriker, 1973); however, this is likely to be a serious over-simplification. For one thing, three Magellanic Cloud sources which are at a distance of $\sim 60 \mathrm{kpc}$ are seen with an intensity of $20 \mathrm{cts} \mathrm{s}^{-1}$. Translated to $9 \mathrm{kpc}$, the distance to the galactic center, these sources would be seen at an intensity of $\sim 900 \mathrm{ct} \mathrm{s}^{-1}$. Even allowing for interstellar absorption, their counting rate would be expected to be at least $500 \mathrm{ct} \mathrm{s}^{-1}$. In fact, only five are seen with such a high counting rate, in spite of the fact that the Milky Way is believed to contain ten times the number of stars as the Magellanic Clouds. The average counting rate of the bright sources in the central region is more like $300 \mathrm{ct} \mathrm{s}^{-1}$. Another difficulty with this idea is that one of the Magellanic Cloud sources, SMC X-1, is apparently in the class of close binary with B supergiant similar to Cyg X-1, Cen X-3, $0900-40$, and $1700-37$ in the Milky Way. However, three of these are in the outer region and none is included among the brighter sources in the central region.

Whether one tries to make them correspond to outer region or bright central region sources, there are too many Magellanic Cloud sources and they are too luminous. Thus, their presence in the Magellanic Clouds may be a reflection of the difference in the average kinds of stars there compared to what is found in the Milky Way or, perhaps more simply, a chance occurrence.

Fortunately, there is a more definite method for determining the space distribution of the central region sources and that is the use of the galactic ridge. As discussed above, this is the excess counting rate, seen along the Milky Way, which may be attributed to unresolved sources. Most recently, Dilworth et al. (1973) have attempted to determine the luminosity function of the X-ray sources using the ridge argument and the actual distribution of the sources. Their conclusion is that very few unresolved sources needed to account for that background. 


\section{Summary}

The distribution of the X-ray sources around the Milky Way is compatible with a dual population. One comprises very young objects, newly born and close to the galactic plane and the other category is, on the average, more distant from the plane of the Galaxy and represents a much older class of stars.

There also appears to be a very large spread in the intrinsic X-ray luminosity of the $\mathrm{X}$-ray sources from at least $10^{35}$ to $10^{38} \mathrm{erg} \mathrm{s}^{-1}$.

\section{Observations of Specific Galactic X-Ray Sources}

We turn now from the general characteristics and distribution of the galactic sources to the properties of those sources, most of which are listed in Table I, for which significant data is available.

\subsection{Cygnus $\mathrm{X}-1$}

In the study of compact galactic X-ray sources, Cygnus X-1 has occupied a central position. It is the source first identified with an optical binary system, and the source most commonly considered as a possible black hole. Cyg X-1 has been observed extensively in the optical and radio frequencies as well as in X-rays, but it is clear that it was the fast-time variability of the X-ray emission which sparked the early
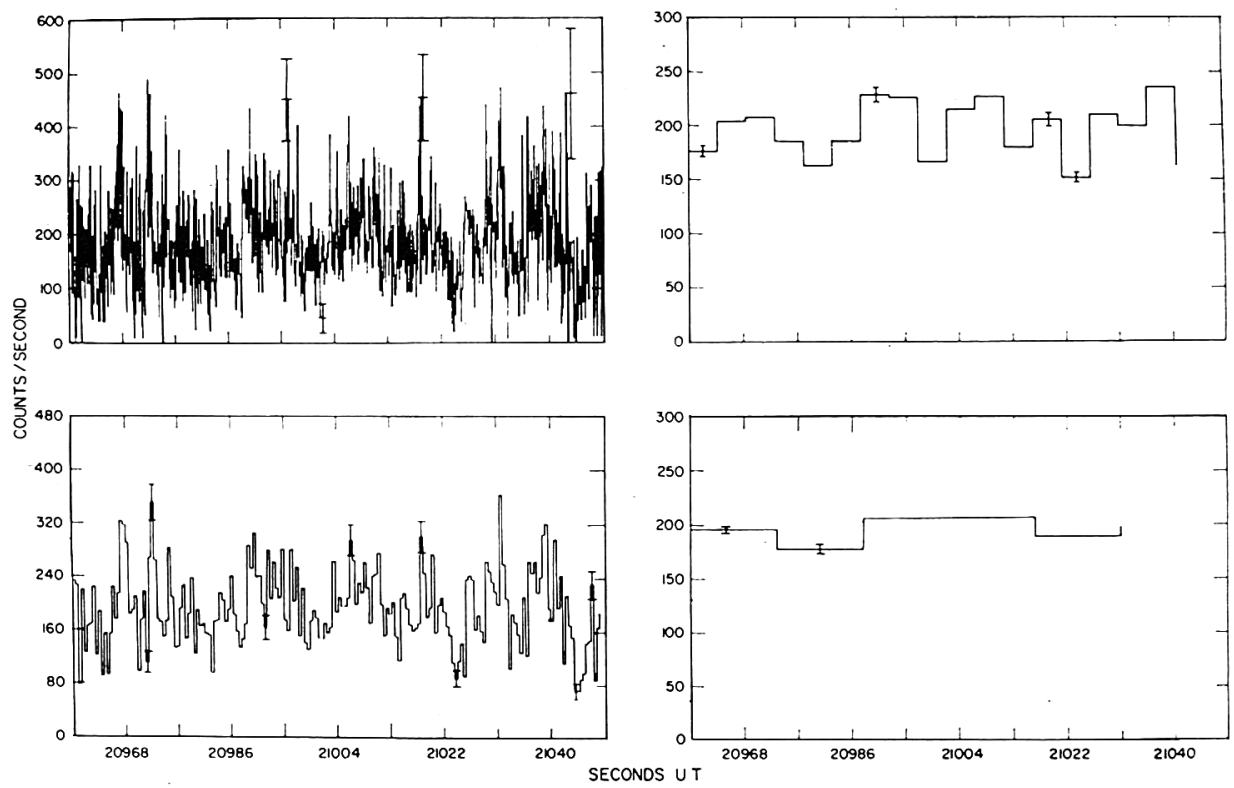

Fig. 3. Observation of Cygnus X-1 on 1971, June 10. Data have been corrected for triangular collimator response. Data are summed over $0.096 \mathrm{~s}, 0.48 \mathrm{~s}$, and $14.4 \mathrm{~s}$ intervals. Typical $1 \sigma$ error bars are shown. 
and continuing interest in the source. Indeed, it was the discovery of this variability in the early Uhuru data which led to the successful search for other variable sources, and to the discovery of the whole class of binary X-ray sources.

The first reports of X-ray variability in Cyg X-1 occurred in the years 1966-1968 and were based on comparison of results from various balloon and rocket flights (e.g., Byram et al., 1966; Overbeck and Tananbaum, 1968). It was in early 1971 that variability on time scales of less than a second was first reported from Uhuru observations (Oda et al., 1971). This variability appeared at times periodic, and led the authors to hypothesize that the X-ray object was a collapsed star - possibly a neutron star or a black hole. This observation was soon followed by reports from other observers presenting varying results, including flaring on a $50 \mathrm{~ms}$ time scale and a 300 ms periodicity (see e.g., Rappaport et al., 1971a; Holt et al., 1971; Shulman et al.,

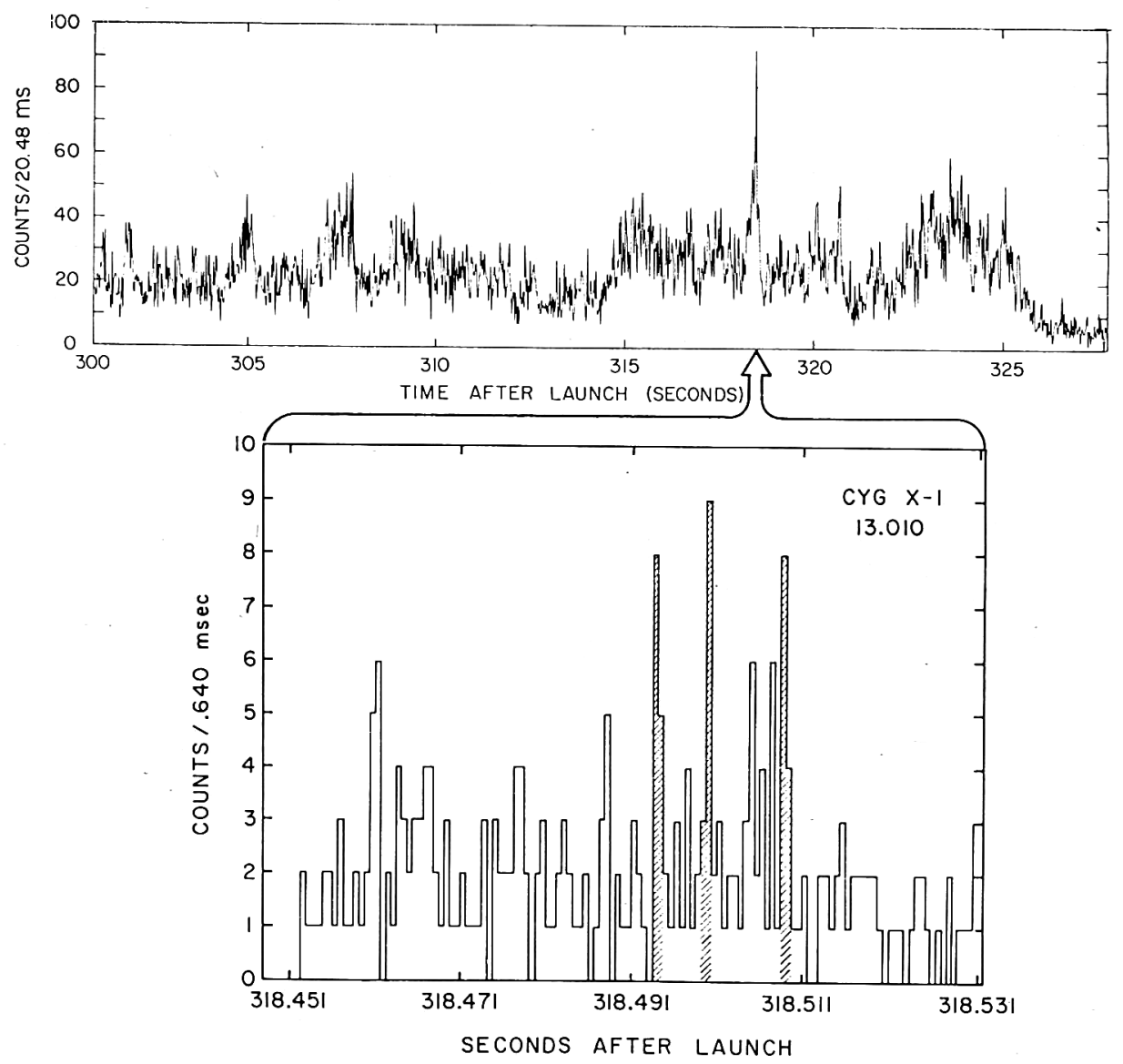

Fig. 4. High time resolution data on Cyg X-1 obtained by Rothschild et al. (1974). The lower portion is an expansion of the 'burst' at $318 \mathrm{~s}$. The crosshatched elements contain almost half the counts of the highest 'spike' within the burst. The distribution of the counts suggests that significant fluctuations occur during a time interval of $1 \mathrm{~ms}$. 
1971). An extensive analysis of six months of Uhuru data by Schreier et al. (1971) and also by Oda et al. (1972) led to the conclusion that pulse trains existed with periods ranging from a few tenths of a second to seconds, but only lasting to some tens of seconds. No single period was consistently present, but significant variability existed on all time scales studied, ranging from a tenth of a second to days (see e.g. Figure 3). The analysis was later extended to include a study of the spectral behavior of these time variations (Brinkman et al., 1974). It was found that although the fluctuations occurred concurrently at both lower (2-5 keV) and higher (5-12 keV) energies, the higher energy pulses were typically narrower. There also appeared to be more power contained in the fluctuations at higher energies.

The range of time variability of Cyg X-1 has recently been dramatically extended by the observation of $1 \mathrm{~ms}$ bursts (Rothschild et al., 1974) as shown in Figure 4. The variability observed leads us to consider a compact object smaller than about $10^{7} \mathrm{~cm}$ as the source of the X-ray emission. This follows from the fact that the emitting region can be no larger than the distance which electromagnetic radiation can travel during the time in which the source intensity changes significantly.

The accurate X-ray location of Cyg X-1 from Uhuru, an MIT rocket flight (Rappaport et al., 1971b), and a Japanese balloon flight (Miyamoto et al., 1971), led to the discovery of a radio source by Braes and Miley (1971) and Hjellming and Wade (1971). This radio source was distinguished by the fact that it was not present up to the end of March 1971. It was later found that the X-ray emission underwent a transition simultaneous with the radio source's appearance (Tananbaum et al., 1972a; Hjellming, 1973). The 2-6 keV flux decreased by a factor of 4, while the $10-20 \mathrm{keV}$ flux increased by 2 as shown in Figure 5. The spectrum before the transition had a low energy excess which could be fitted by either a power law with energy index of 4 , or an exponential with a temperature of $11 \times 10^{6} \mathrm{~K}$ (Schreier et al., 1971; Tananbaum et al., 1972). The disappearance of this low energy component as the radio source appeared may indicate a decrease in the plasma density, reducing the $\mathrm{X}$-ray emission measure and lowering the plasma frequency or the free-free selfabsorption of the radio emission.

The precise error box determined for the radio source $\left(\$ 1^{\prime \prime}\right)$ contained the star HDE 226868. This star was found to be a 5.6 day spectroscopic binary (Webster and Murdin, 1972; Bolton, 1972). The star appears as a 9th magnitude B0Ib supergiant, at a minimum distance of $2.5 \mathrm{kpc}$ (Bregman et al., 1973; Margon et al., 1973). The primary thus has a mass of about $30 \mathfrak{M}_{\odot}$, leading to a mass $\gtrsim 6 \mathfrak{M}_{\odot}$ for the X-ray source. Even if the primary consists solely of a helium burning core, the secondary mass is still $\gtrsim 3 \mathfrak{M}_{\odot}$ (Van den Heuvel and Ostriker, 1973). Analysis of $\sim 40$ days of continuous data from Uhuru showed no evidence of X-ray intensity variation correlated with the 5.6-day period (Tananbaum et al., 1972a); however, the Copernicus data do show a small, gradual decrease during a six day observing run (Sanford et al., 1974) centered on the phase of closest approach of the optical star to the Earth. Larger decreases on a shorter time scale $(\sim 30 \mathrm{~min})$ have been reported at the same phase (Sanford, 1973; Li and Clark, 1974). Thus, while there is some evidence for 

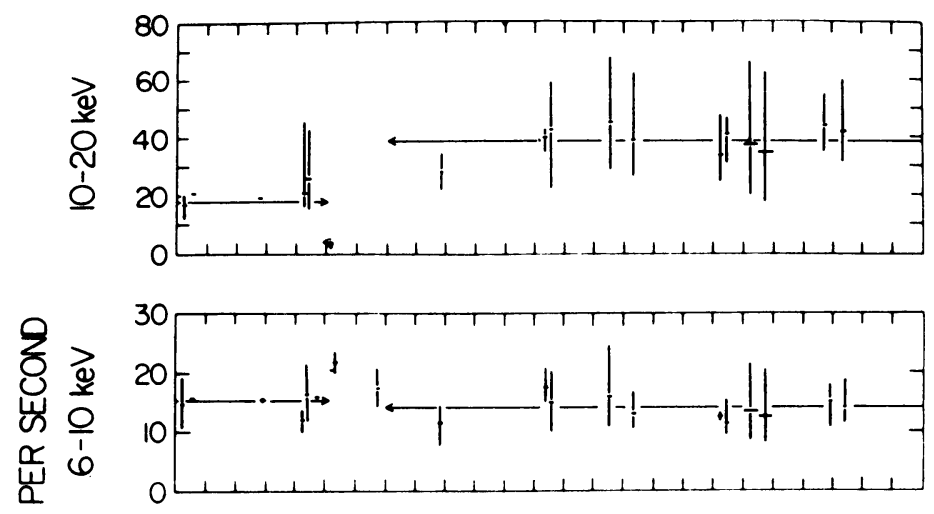

5
$\frac{5}{3}$
0
$\frac{0}{2}$
$\frac{1}{x}$
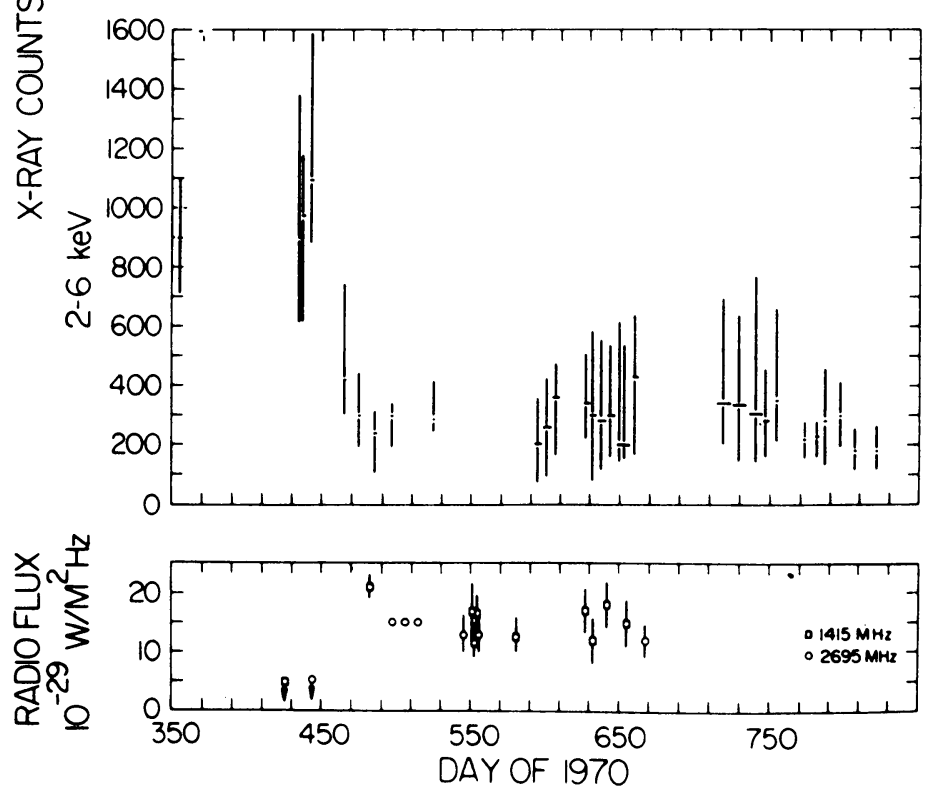

Fig. 5. Sixteen months of observations of Cygnus X-1. X-ray data are shown for three energy bands, 2-6 keV, 6-10 keV, and 10-20 keV plotted vs. day of 1970 . The transition discussed in the text occurred in the period near day 450 . The radio data are shown at the bottom of the figure.

a correlated effect, there is no evidence of an eclipse, which limits the orbital inclination with respect to our line of sight. It is the high mass necessitated for the $\mathrm{X}$-ray source combined with its compact nature which leads to the consideration of Cyg X-1 as a black hole.

We can summarize three main points leading to the identification of Cyg X-1 as a black hole: (1) HDE 226868 is the optical counterpart of the X-ray source, (2) the mass of HDE 226868 is greater than $20 \mathfrak{M}_{\odot}$, and (3) the X-ray emitting object is compact. The identification of HDE 226868 with Cyg X-1 is well established via the positional coincidence of the optical and radio along with the correlated intensity 
variation of the X-ray and radio. Further evidence is given by the possible 5.6 day effect in soft X-rays, and also by the fact that other X-ray binaries have also been identified with BOI supergiants.

The large mass of the primary has also survived extensive critical discussion. The original conservative estimate of a primary mass of greater than $20 \mathfrak{M}_{\odot}$ was based on the assumption that the star was a normal BOIab supergiant according to its spectral characteristics. This was criticized by Paczynski (1973) and by Trimble et al. (1973), based on the fact that the spectrum is indicative of only the surface gravity and temperature of a star. Thus, one could create a low mass model of lower luminosity which mimics the spectrum of a B0 supergiants. This, however, was ruled out by the distance measurements already mentioned. It was also noted that the proximity of the strong X-ray source might alter the appearance of the spectrum. However, no reflection effect is observed, nor for that matter is one really predicted; the optical variations are consistent with tidal distortion, which in turn helps determine the mass function (see e.g. Mauder, 1973). Further evidence for the non-anomalous appearance of the optical spectrum comes from the normal spectrum of SMC X-1, whose distance is known (see below). Thus it appears very likely that the $\mathrm{X}$-ray object does indeed have a mass of at least $6 \mathfrak{M}_{\odot}$.

The X-ray variability leaves little doubt as to the compact size of the X-ray emitting region. The only alternative to a compact star is the presence of active regions on or around a normal star. However, a main sequence star of $6 \mathfrak{M}_{\odot}$ should produce appreciable visible light in the system, both by emission and by reflection from the primary. No such contribution is seen. Furthermore, there is no obvious X-ray emission mechanism in this case.

In conclusion, there appears strong evidence that the $\mathrm{X}$-ray object is compact with a mass significantly greater than $3 \mathfrak{M}_{\odot}$. It is impossible to have a neutron star this massive (Ruffini, 1973) and it has not been demonstrated how a differentially rotating white dwarf could be stable and could emit the observed X-rays. However, an accretion disk around a black hole is consistent with all observations thus far. A modest accretion rate of $\sim 10^{-9} \mathrm{M}_{\odot} \mathrm{yr}^{-1}$ can produce the X-ray luminosity. Integration over the entire disk can produce the observed power law spectrum, and irregularities in the accretion disk can produce the observed irregular variability with time scales down to a millisecond.

\subsection{Centaurus $\mathrm{X}-3$}

Centaurus X-3 was found to be a binary X-ray pulsar in the wake of the discovery of the time variability of Cygnus X-1 (Giacconi et al., 1971; Schreier et al., 1972a). Cen X-3 pulsates with a period of $4.8 \mathrm{~s}$, a stable period in contrast to the erratic variability of Cyg X-1, and in addition shows eclipsing behavior in the X-rays. Furthermore, the measurement of the Doppler variation of the pulsations established that the source was in a massive binary system.

The first and most obvious characteristic of Cen $\mathrm{X}-3$ is its regular pulsations. Figure 6 shows $100 \mathrm{~s}$ of $2-6 \mathrm{keV}$ counting rate data along with a minimum chi-square 


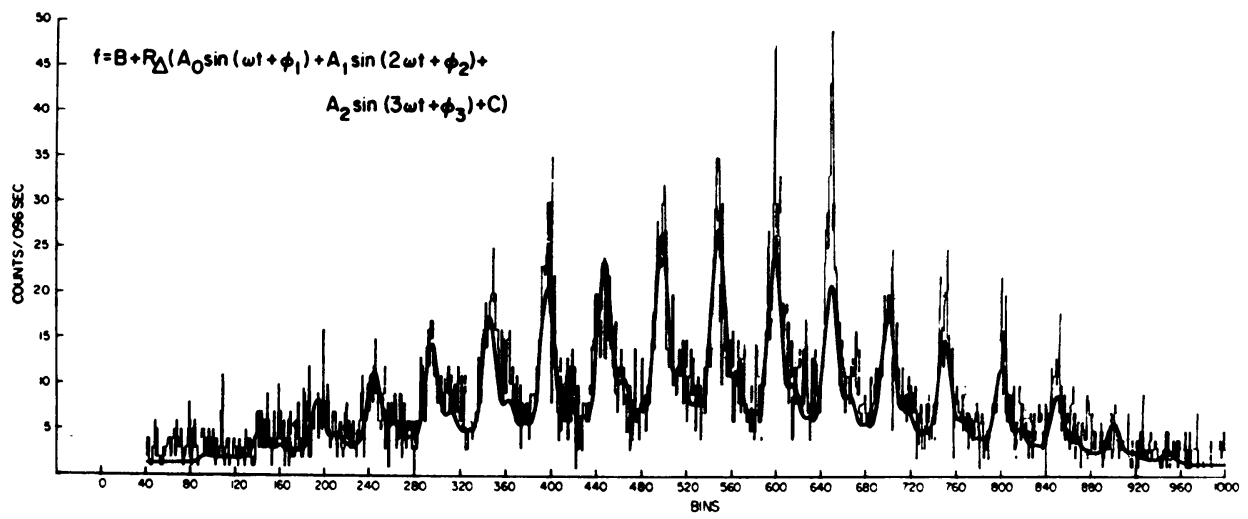

Fig. 6. Counts accumulated in 0.096-s bins from Cen X-3 during a 100-s pass in 1971, 7 May. The functional fit obtained by minimizing $\chi^{2}$ is shown as the heavier curve.
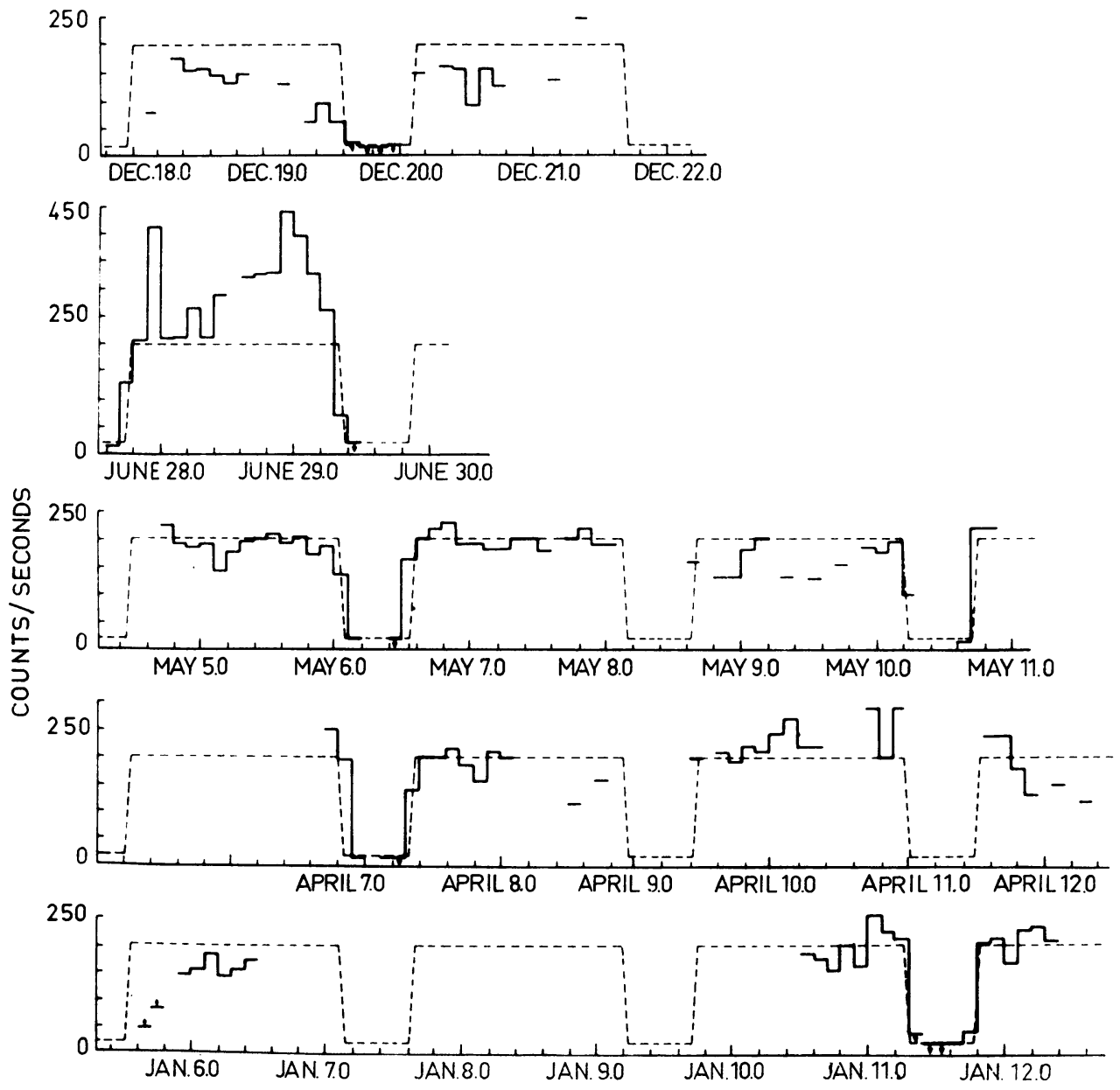

Fig. 7. Observations of Cen $\mathrm{X}-3$ by Uhuru showing regularity of eclipse. 
fit of a sinusoid with harmonics. The X-rays are seen to be over $70 \%$ pulsed with a $4.8 \mathrm{~s}$ period, although the shape of the pulse has been found to vary.

The binary nature of Cen X-3 is illustrated in Figure 7 where observations of the source during 1971 are shown along with an average light curve. The orbital period is 2.09 days, with an eclipse duration of 0.49 days, and with the transition into and out of eclipse taking some 0.04 days. The intensity during eclipse does not reach zero, and it can also be seen that the average non-eclipse intensity is quire variable. This variability in the average (over pulsations) intensity extends to the existence of extended low states, periods of time when the source is seen very weakly, and to times when the intensity alternates erratically between high and low states. No recurrent behavior has yet been observed for the extended lows, although the Uhuru data suggest time scales of 2 to 4 months for the durations of the states.

The Uhuru data has recently yielded a transition from a low to a high state which is illustrated in Figure 8. As can be seen, the source comes on first within a restricted range of orbital phase, gradually broadening and increasing in intensity until it reaches its full intensity and width, several binary periods later. The $4.8 \mathrm{~s}$ period, although stable to better than $10^{-6}$ over time scales of at least days, has been observed to change over months. The net change in the period observed during 1971-72 was a decrease of approximately $1.5 \mathrm{~ms} \mathrm{yr}$, i.e., $\dot{p} / p \cong-3 \times 10^{-4} \mathrm{yr}^{-1}$. The changes in the period are definitely not uniform and probably not monotonic. A possible correlation between the pulsation period changes and the extended lows as determined from Uhuru data is shown in Figure 9; the period is shown on top and a characterization of the average intensity state is shown on the bottom. It can be noticed that the large changes in period occurred during intervals of time in which extended lows also occurred. If this correlation proves to be real, it may relate the spin rate changes of the pulsar to the absorption of X-rays via an accretion model.

The orbital period of Cen X-3 has also been found to change, as shown in Figure 10. This change is seen to be non-linear and in fact, to change sign. It thus cannot be explained by simple mass loss from the system, but may be due to competing massloss, mass-exchange effects or possibly to apsidal motion.

The energy spectrum of Cen X-3 in the X-ray range is typically hard. There is a variable low energy cutoff ranging from 1.5 to $4 \mathrm{keV}$ followed by a flat spectrum to $20 \mathrm{keV}$. Above $\sim 25 \mathrm{keV}$, there is an exponential decrease (Baity et al., 1974). There is also spectral variability across the $4.8 \mathrm{~s}$ pulse, with the peak intensity being harder.

Cen X-3 has recently been identified with a 13th magnitude $\mathrm{B} 0$ supergiant by Krzeminsky (1973). The distance is thought to be about $10 \mathrm{kpc}$. The X-ray observations had previously predicted the existence of such a massive star in the system (Schreier et al., 1972a); analysis of the Doppler variations of the pulsations had determined the orbital radius projected into our plane of view as $1.10 \times 10^{12} \mathrm{~cm}$, the projected orbital velocity of the X-ray source as $415 \mathrm{~km} \mathrm{~s}^{-1}$, and the mass function as $15.4 \mathfrak{M}_{\odot}$. This last is of course a lower limit on the mass of the optical companion. 


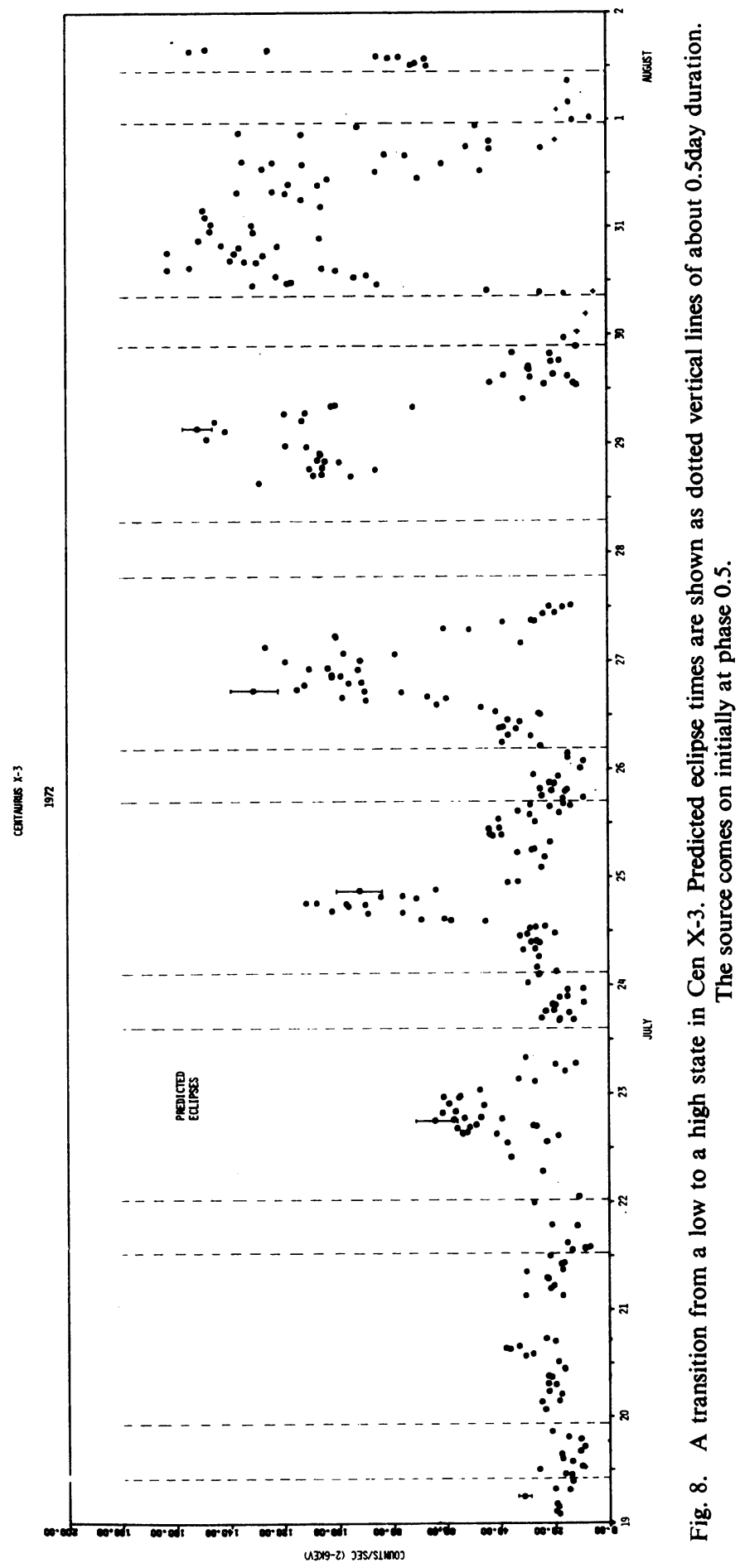




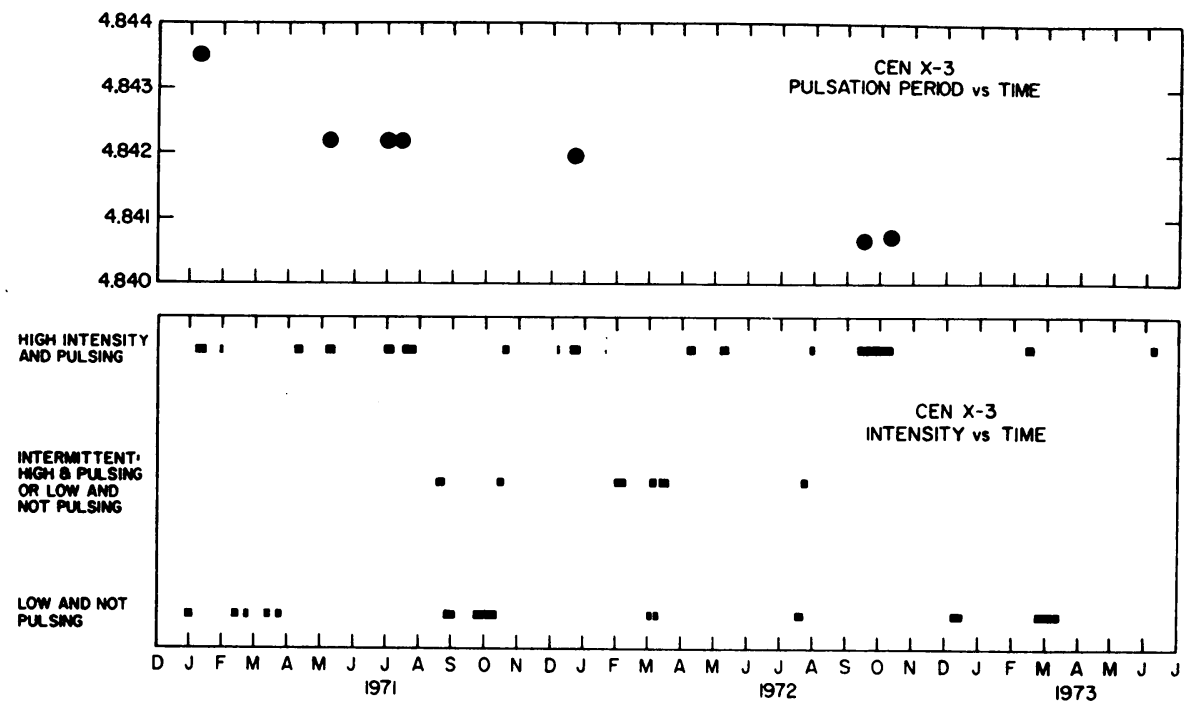

Fig. 9. Top: Heliocentric period of Cen X-3. Bottom: Long term intensity states of Cen X-3.

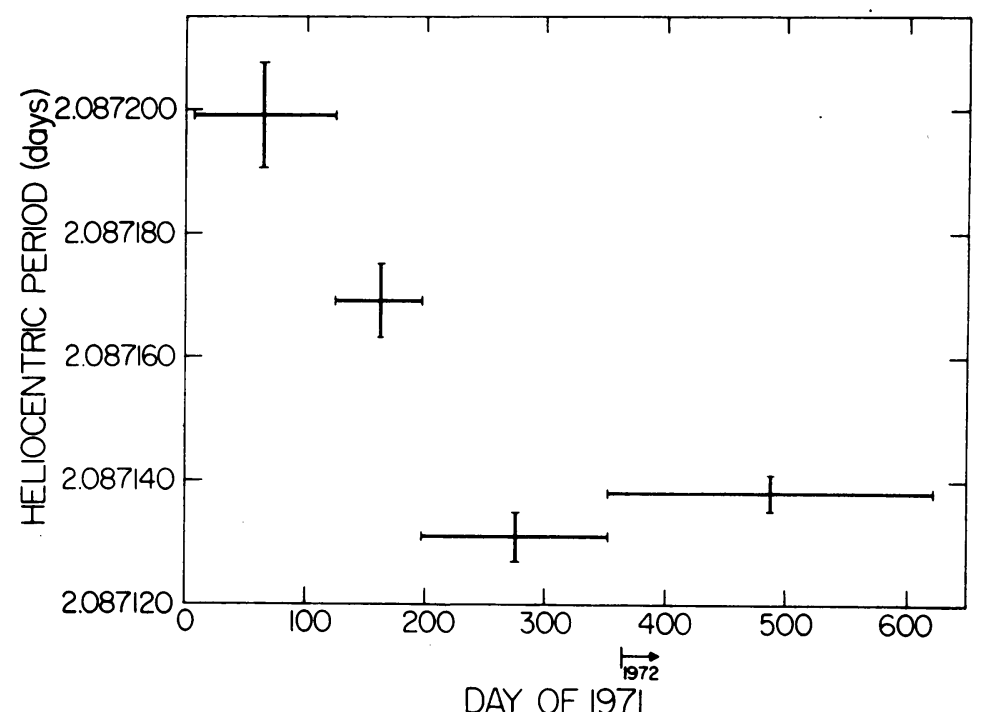

Fig. 10. Variation in the orbital period of Cen X-3.

\subsection{Hercules $X-1$}

The X-ray source Hercules X-1 was discovered in the Uhuru data just as Cen X-3 was recognized as an X-ray pulsar in a binary system (Tananbaum et al., 1972b). Since the two sources' $X$-ray characteristics are qualitatively the same, it was straightforward to interpret the Her X-1 observations in the framework of a binary model. Helped by its early identification with an optically variable star of the same period, Her X-1 has become the most widely studied compact X-ray source. Many of the 
observations are explained in terms of a mass transfer binary system, in which a rotating neutron star is accreting matter from a companion. The amount of data compiled on this source along with the theoretical work done makes it impossible to review completely here. However, we will attempt to summarize both the main observational characteristics of the source and the basic theoretical considerations.

There are three periodicities observed in the Her X-1 X-ray data: a pulsation period of $1.24 \mathrm{~s}$, an orbital period of 1.7 days, and an approximate 35-day on-off cycle. There is yet a fourth kind of variability on a time scale of years, discovered through the study of historical material on the optical companion. The short period pulsations, as shown in Figure 11, are similar to those of Cen X-3. The pulse shape has been studied in some detail (Doxsey et al., 1973; Giacconi et al., 1973; Holt et al., 1974). It is variable both from pulse to pulse and on the average over minutes, sometimes appearing as a main pulse and an interpulse, and sometimes as a double peaked main pulse. Like Cen X-3, the pulsation period is stable over days, except for the binary Doppler effect, but shows changes on the order of microseconds from month to month as shown in Figure 12. A net decrease of $6 \mu \mathrm{s}$ in $1.25 \mathrm{yr}$ was observed, corresponding to $\dot{p} / p \cong-4 \times 10^{-6} \mathrm{yr}^{-1}$.

As shown in Figure 13, the 1.7-day orbital periodicity is observed both in the X-ray intensity and in the Doppler variations of the pulsation period. The 2-6 keV flux goes from about $100 \mathrm{cts} \mathrm{s}^{-1}$ to below a few counts per second. The transitions between high state and eclipse take less than $12 \mathrm{~min}$, and the eclipse lasts for 0.24 days. No

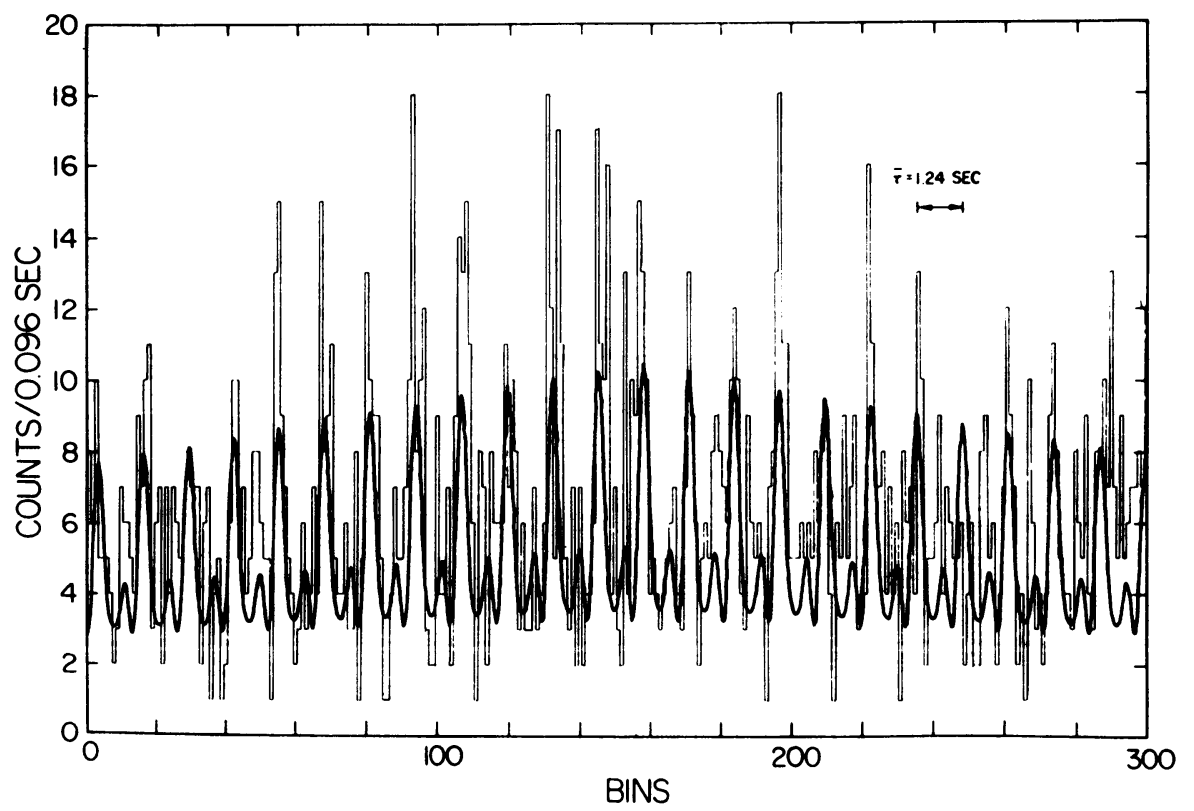

Fig. 11. Counts accumulated in 0.096-s bins from Hercules X-1 during the central $30 \mathrm{~s}$ of a 100-s pass in 1971, November, 6 . The heavier curve is a minimum $\chi^{2}$ fit to the pulsations of a sine function, its first and second harmonics plus a constant, modulated by the triangular response of the collimator. 


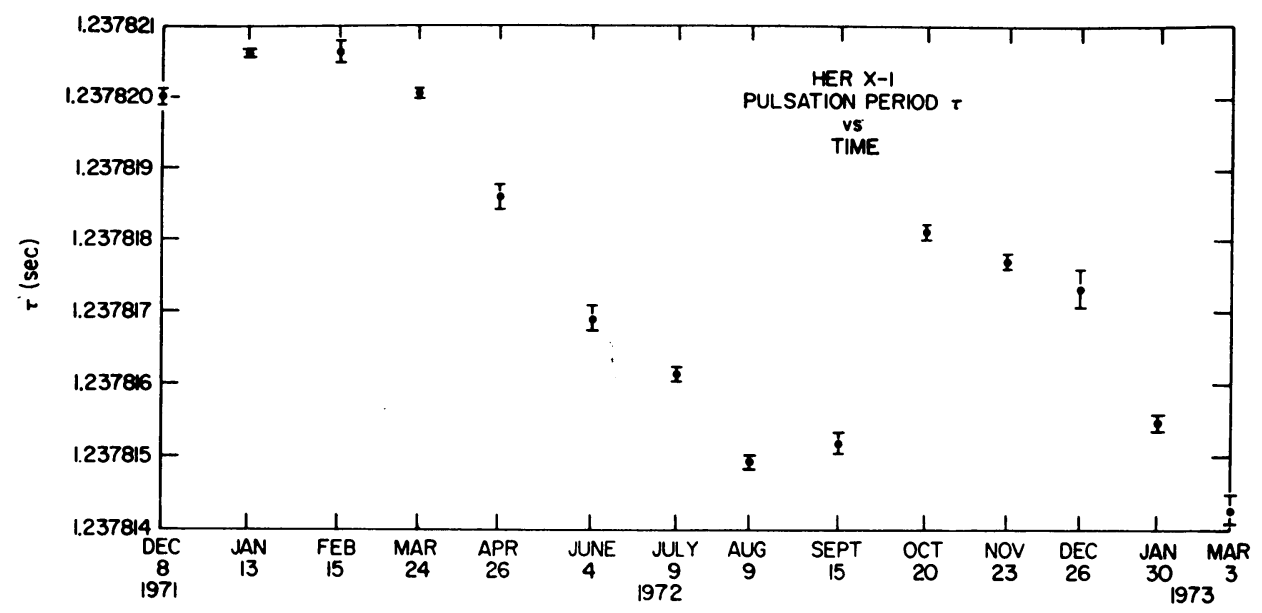

Fig. 12. The Her X-1. heliocentric period determined for the 1.2-s pulsations during 14 high states from 1971 to 1973.

overall orbital period change has been seen in the X-ray data over more than a year to a part in $10^{6}$. We can not exclude changes over several months of order $10^{-5}$ days. The orbital period of the system is also seen in visible light. Following an improved X-ray position by Clark et al. (1972) and the observation by Liller (1972a) that the star HZ Herculis had a large UV excess, Bahcall and Bahcall (1972) and Liller (1972b) discovered 1.7-day light variations of that star. The optical variations of 1.5 mag. are in phase with the X-rays, as seen in Figure 14, suggesting X-ray heating of the close side of the optical companion as the cause (Forman et al., 1972). Spectroscopic observations are complicated by the heating; temperatures of $10^{4} \mathrm{~K}$ and $7 \times 10^{3} \mathrm{~K}$ respectively are estimated for the two sides. The spectral type of $\mathrm{HZ} \mathrm{Her}$ is late A or early F; the distance estimates range from 2 to $6 \mathrm{kpc}$. The optical light curve near minimum intensity is much narrower than the $\mathrm{X}$-ray eclipse. This has been explained variously as (1) deep convective transport of the absorbed radiation (Wilson, 1973), (2) different sources of the optical light including emission from an accretion disk around Her X-1 (Boynton et al., 1973; Crampton and Hutchings, 1972; Strittmatter et al., 1973; Basko and Sunyaev, 1973), and (3) radiation from above the photosphere of HZ Her (Joss et al., 1973).

Optical pulsations from HZ Her have been definitely detected on occasion (Lamb and Sorvari, 1972; Davidsen et al., 1972; Middleditch and Nelson, 1973; Groth et al., 1973). They are never more than $0.2 \%$ of the total light, and period measurements indicate that they may be emitted from gas moving in the binary system rather than from $\mathrm{HZ}$ Her.

In addition to the pulsations and the orbital period, there is the third cycle. The source is seen to turn on about every 35 days. It then follows a regular pattern, decreasing to below detectable limits after about 11 days and remaining low for 24 days. Three such 'on-states' are shown in Figure 15. Uhuru-observations have 


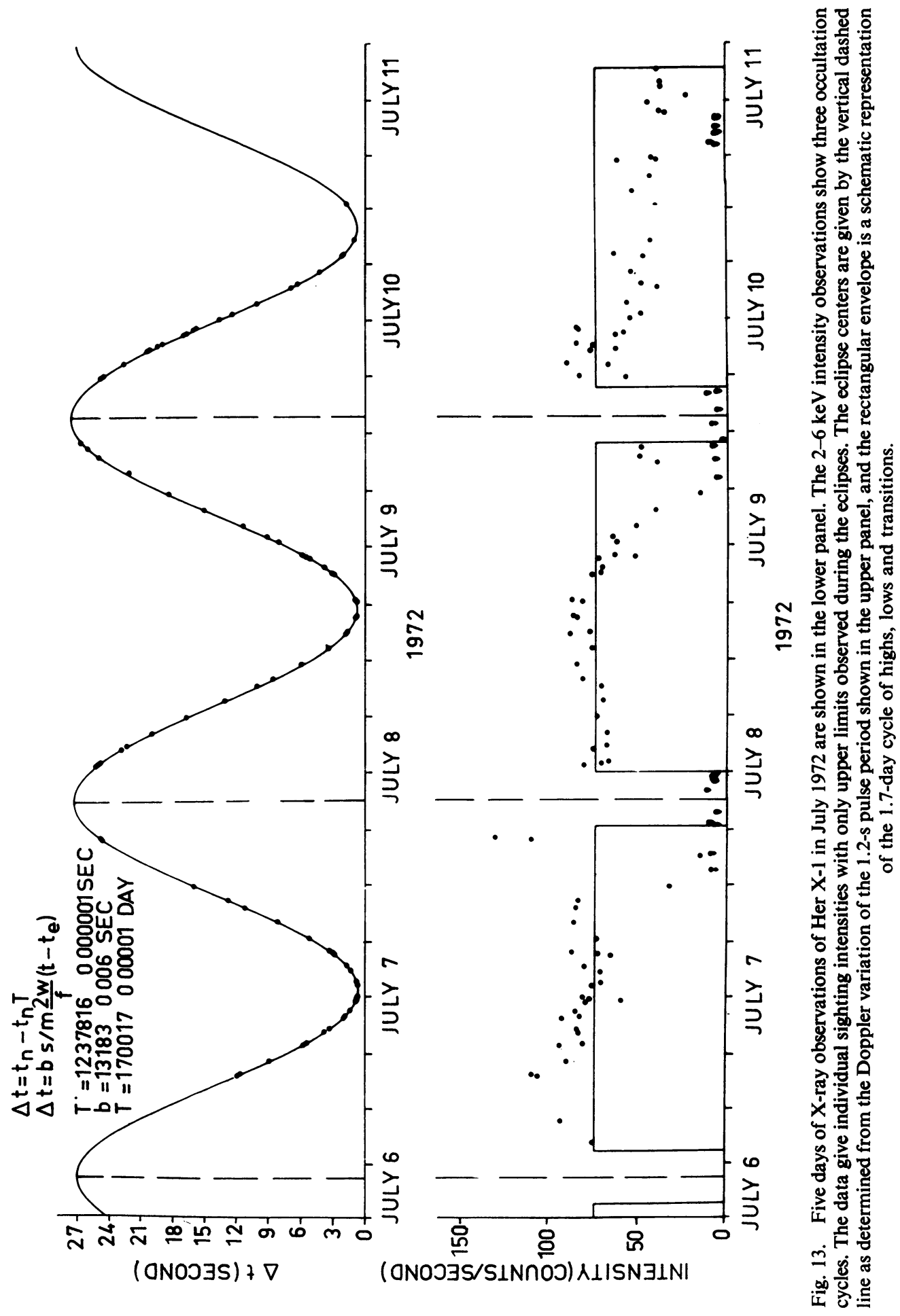




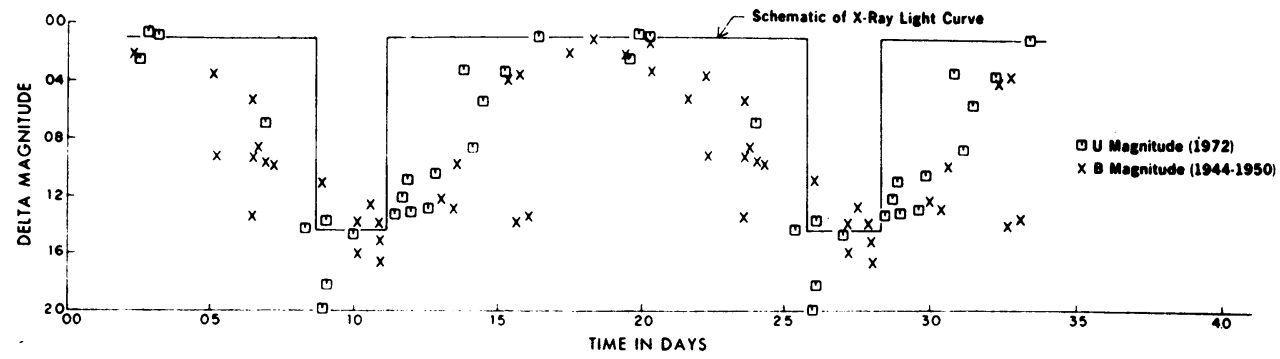

Fig. 14. Optical observations of $\mathrm{HZ}$ Herculis folded with 1.70015 days as a period. Squares indicate 1972 U magnitude observations and $x$ 's give $B$ magnitude observations from plates taken in 1944 1950. The optical curve shows much more sinusoidal variation than the rectangular 1.7-day X-ray light curve which is also indicated. Observe that the phase of the optical minimum coincides with the center of the X-ray eclipse.

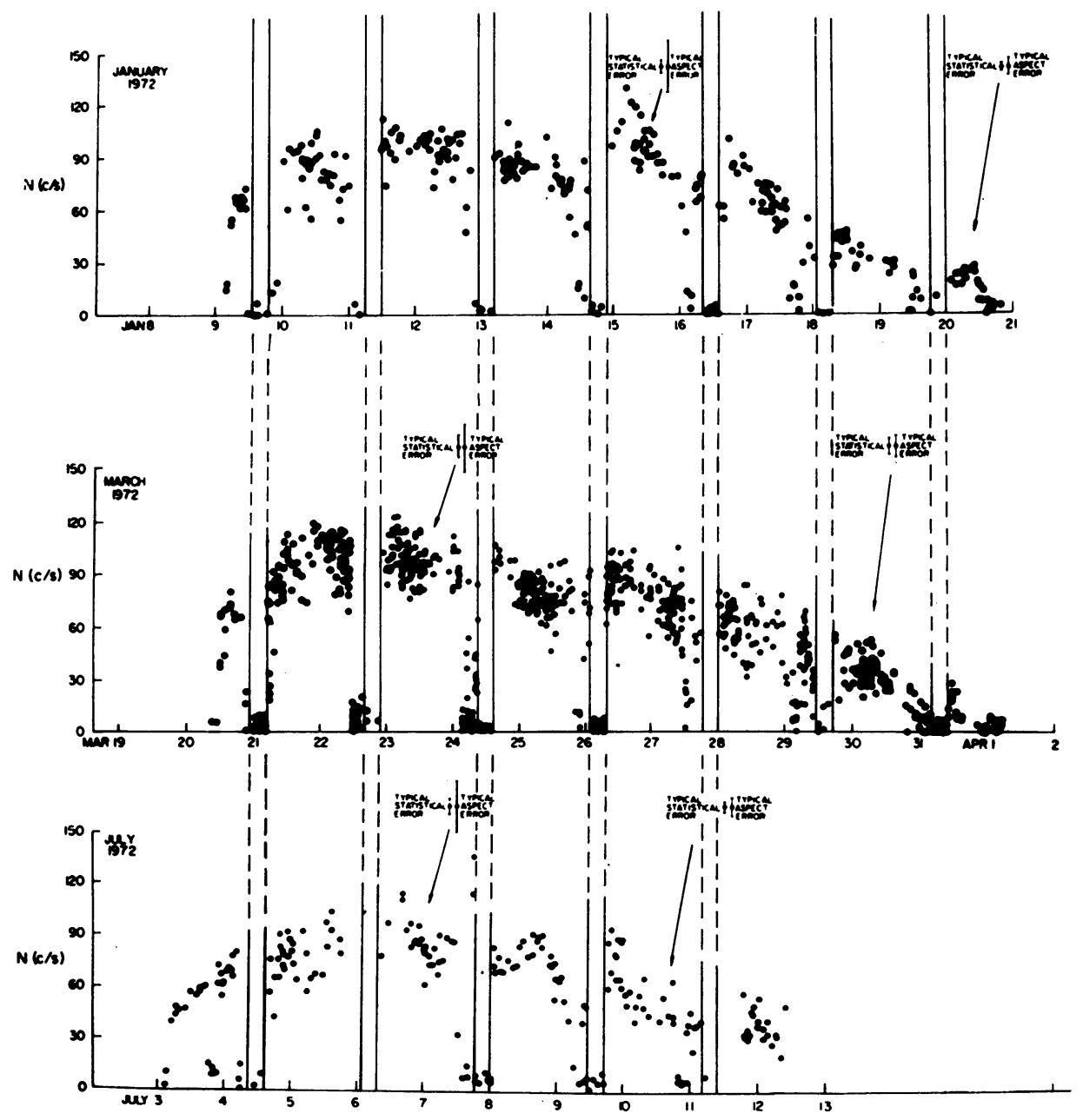

Fig. 15. Detailed 2-6 keV intensity observations of Her X-1 during January 1972, March 1972, and July 1972 high states. Each individual sighting of the source is represented by a dot with typical statistical and systematic errors indicated. The curves all show the sharp turn-on, the broad maximum, the gradual decrease over several days, and the presence of the dips discussed in the text. An example of a dip clearly separated from the eclipse can be seen on July 10.9 . 
never shown any violations of this general cycle; on 67 of the 96 days in four 'offstates' as well as on scattered other 'off' days the source was not detected. However, a recent observation from the Copernicus X-ray experiment (Fabian et al., 1973) reported emission during one 'off-state'.

Other features of the X-ray emission can also be seen in the figure. The turn-ons in the 35-day cycle are seen to be sharp. As is illustrated in Figure 16, they all tend to occur near two orbital phases 0.2 and 0.7 . Thus, within the approximate 35-day cycle, the actual turn-ons are separated by typically 34.0-, 34.85- or 35.7-day intervals. Although there appears to be an underlying clock, it only keeps correct time on the average, and the turn-ons themselves are orbit phase dependent. Another important feature apparent in the figure are the intensity dips. In addition to the regular eclipses and the inherent variability of the source, additional recurring dips are seen. Although the average duration of the dips is comparable to that of an eclipse, both the intensity in a dip and the duration are quite variable. Furthermore, the dips regularly move with respect to the 1.7-day orbital phase, appearing progressively earlier before each succeeding eclipse in a given 11-day 'on-state'. Additional dips have been seen about 0.6 days after turn-on when the turn-on occurred at orbital phase 0.2 . The X-ray spectrum during the dips as well as during turn-on shows a significant low energy
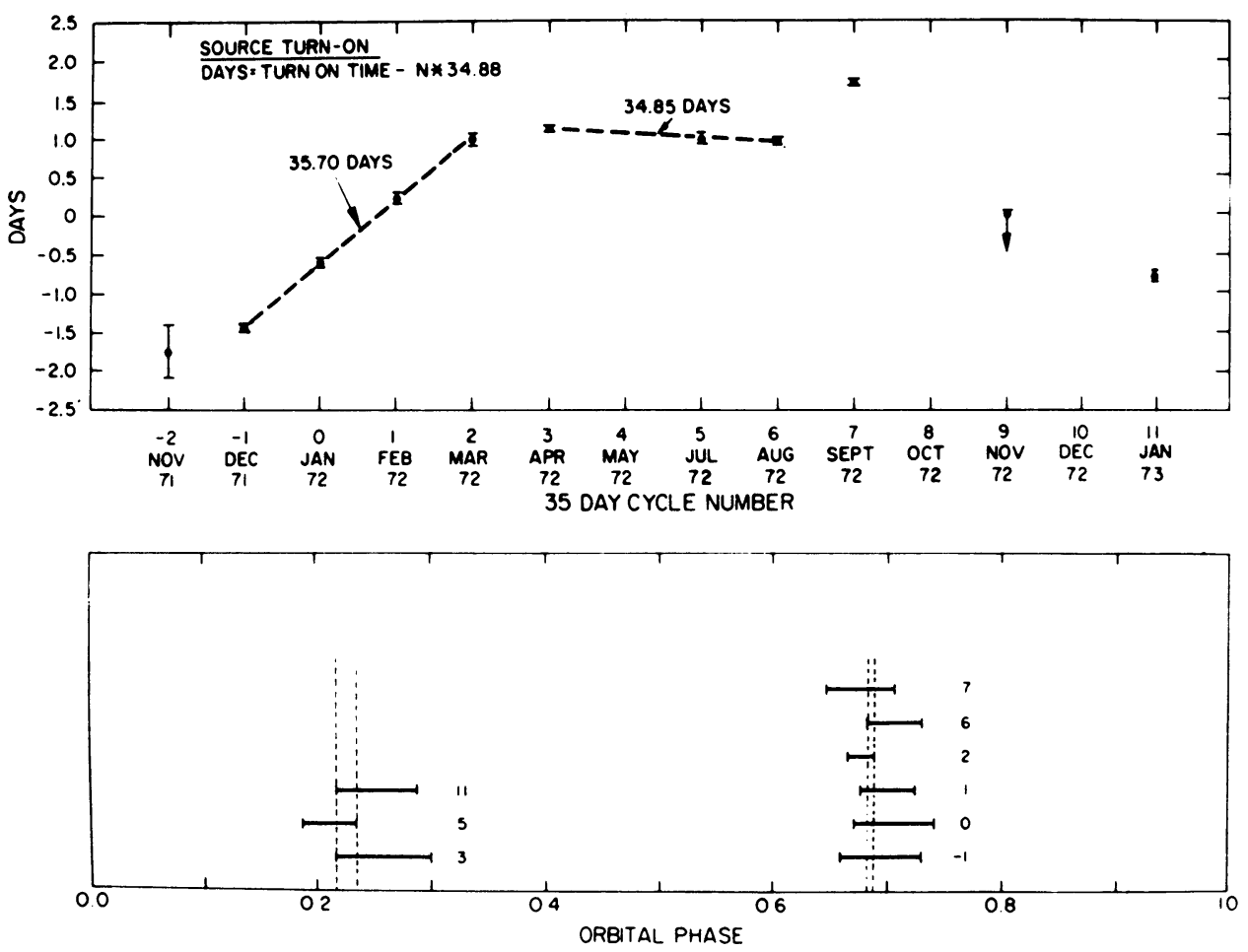

Fig. 16. Turn-on time and orbital phase of Her X-1 X-rays. Time between turn-ons is not fixed, however, as seen in the lower box, the turn-ons occur at only two orbital phases. 
cutoff of about $4 \mathrm{keV}$ implying absorption of the X-rays. This is to be compared with a cutoff of $1.5 \mathrm{keV}$ or less during the rest of the on-state. It should be noted that the $4 \mathrm{keV}$ cutoff implies $\sim 10^{12} \mathrm{H}$ atoms $\mathrm{cm}^{-3}$ in a region of order $10^{11} \mathrm{~cm}$. Above $4 \mathrm{keV}$ the spectrum is quite flat extending beyond $20 \mathrm{keV}$, where it decreases abruptly (Ulmer et al., 1972a).

The optical behavior correlates with this 35-day cycle. The light curve shown in Figure 16 is known to vary continuously and systematically during this time interval. As shown in Figure 17, taken from Boynton et al. (1973), the light is most peaked at the beginning of the 35-day cycle when the X-rays are off and broadens when the

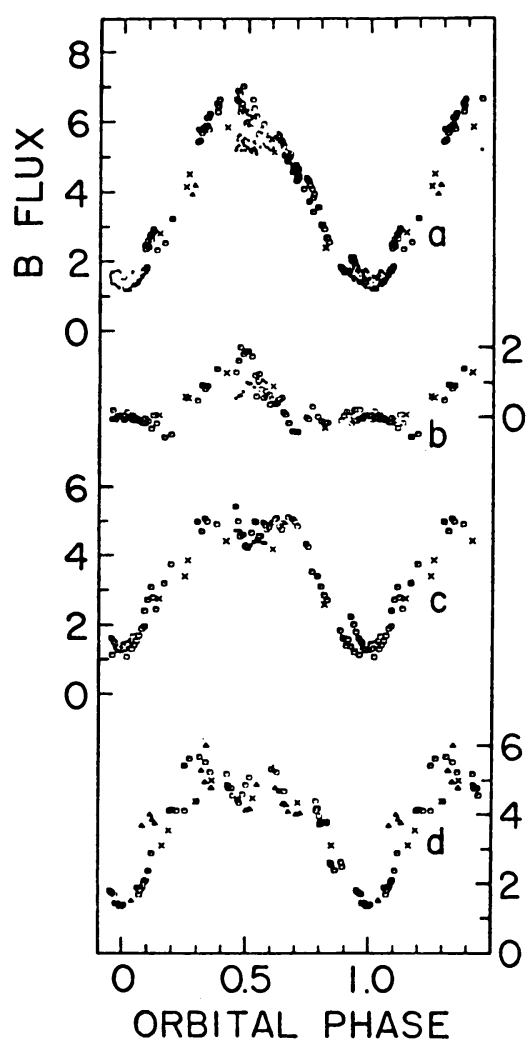

Fig. 17. Variations in the light curve from $\mathrm{HZ}$ Her from Boynton et al. (1973). Upper curve (a) is obtained when X-rays are off lower curve, (b) when X-rays are on. The light curves show systematic variations through the 35-day cycle between the two.

$\mathrm{X}$-rays come on. At all times the peak of the visible light coincides with the peak of the X-rays. More extensive measurements performed by Chevalier and Ilovaisky (1974) confirm this behavior. The fact that the optical emission is essentially undiminished when the X-rays are off, means that the system is continuously powered during the 35 days. This optical behavior has been seen in historical material as far back as 1890 (Jones et al., 1973; Cherepashchuk et al., 1973). However, it has been 
found, that on a time scale of years (most recently from 1949-1956), the optical variability disappears which presumably means that all activity ceased.

The mass of Her X-1 is of special interest, since it is thought to be the first observed neutron star in a binary system. As with Cen X-3, the Doppler variations of the pulsar period allow for an accurate determination of the projected orbital radius $\left(3.95 \times 10^{11} \mathrm{~cm}\right)$, velocity $\left(169.0 \mathrm{~km} \mathrm{~s}^{-1}\right)$, and mass function

$$
\frac{\mathfrak{M}^{3} \sin ^{3} i}{\left(\mathfrak{M}+\mathfrak{M}_{x}\right)^{2}}=1.69 \times 10^{33} \mathrm{gm}=0.85 \mathfrak{M}_{\odot} \text {. }
$$

Unfortunately, further information is needed to determine $\mathfrak{M}_{x}$ itself. The determination of both the orbital velocity and the spectral type of $\mathrm{HZ} \mathrm{Her} \mathrm{which} \mathrm{would} \mathrm{give}$ the primary mass are complicated by the X-ray heating; current best values are 1.5-2.5 $\mathfrak{M}_{\odot}$. The inclination is estimated to be $>85^{\circ}$ from the optical light curve. The X-ray mass is then found to be between 0.5 and $1.8 \mathfrak{M}_{\odot}$. Another mass determination technique relates the mass ratio to the size of the Roche lobe and to the size of the occulting region. Using just the $\mathrm{X}$-ray data then gives (for an inclination of $85^{\circ}$ ) masses of 1.04 and $1.99 \mathfrak{M}_{\odot}$ for the X-ray source and $\mathrm{HZ}$ Her respectively.

The observations of Her X-1-HZ Her can be qualitatively explained by a model invoking an oblique rotating magnetic neutron star in orbit about $\mathrm{HZ} \mathrm{Her.} \mathrm{The}$ presence of a compact object other than a black hole is required to produce the regular pulsations. The presence of a gas stream and also an accretion disk is suggested by the presence and regular advance of the absorption dips. Accretion of matter onto the neutron star can easily power the X-ray luminosity of $10^{37} \mathrm{erg} \mathrm{s}^{-1}$. The funneling of the matter by an oblique rotating magnetic dipole can explain the pulsations. The 35-day cycle can be caused either by cessation of mass transfer from the primary or by cessation of accretion onto the secondary, due for example to precession of the magnetic poles out of the accretion disk (see e.g., Lamb et al., 1973). Although many aspects of the system are not yet explained, and there is far from universal acceptance of this model, it remains the most consistent and most detailed explanation of the Hercules system.

\subsection{Cygnus $\mathrm{X}-3$}

The source Cygnus X-3 represents yet another mode of behavior, not yet seen in any other X-ray sources. Its intensity varies rather smoothly with a 4.8 -h period, quite differently from the eclipsing binaries. It also is identified with a flaring radio source and a 4.8-h periodic infrared object.

The basic X-ray behavior is shown in Figure 18 where the 2-6 keV Uhuru observations of Cyg X-3 for 9 days in May are plotted (Parsignault et al., 1972). The intensity is seen to vary by a factor of two with a period of $4.8 \mathrm{~h}$. The behavior is essentially independent of energy from below $2 \mathrm{keV}$ to $10 \mathrm{keV}$; thus, the light curve can not be due to photoelectric absorption which would preferentially attenuate low energies $\left(\sim \exp \left\{-(E a / E)^{8 / 3}\right\}\right)$. A single period of $0.199681 \pm 0.000002$ days is consistent with all data from December 1970 through May 1972. There is evidence for 
variability in the shape of the light curve, both on short time scales and on the average (Canizares et al., 1973), making it difficult to ascertain period changes.

The average intensity of Cyg X-3 has also varied in the course of two years of observations. Intensity levels as averaged over the 4.8 - $h$ cycle ranging over a factor
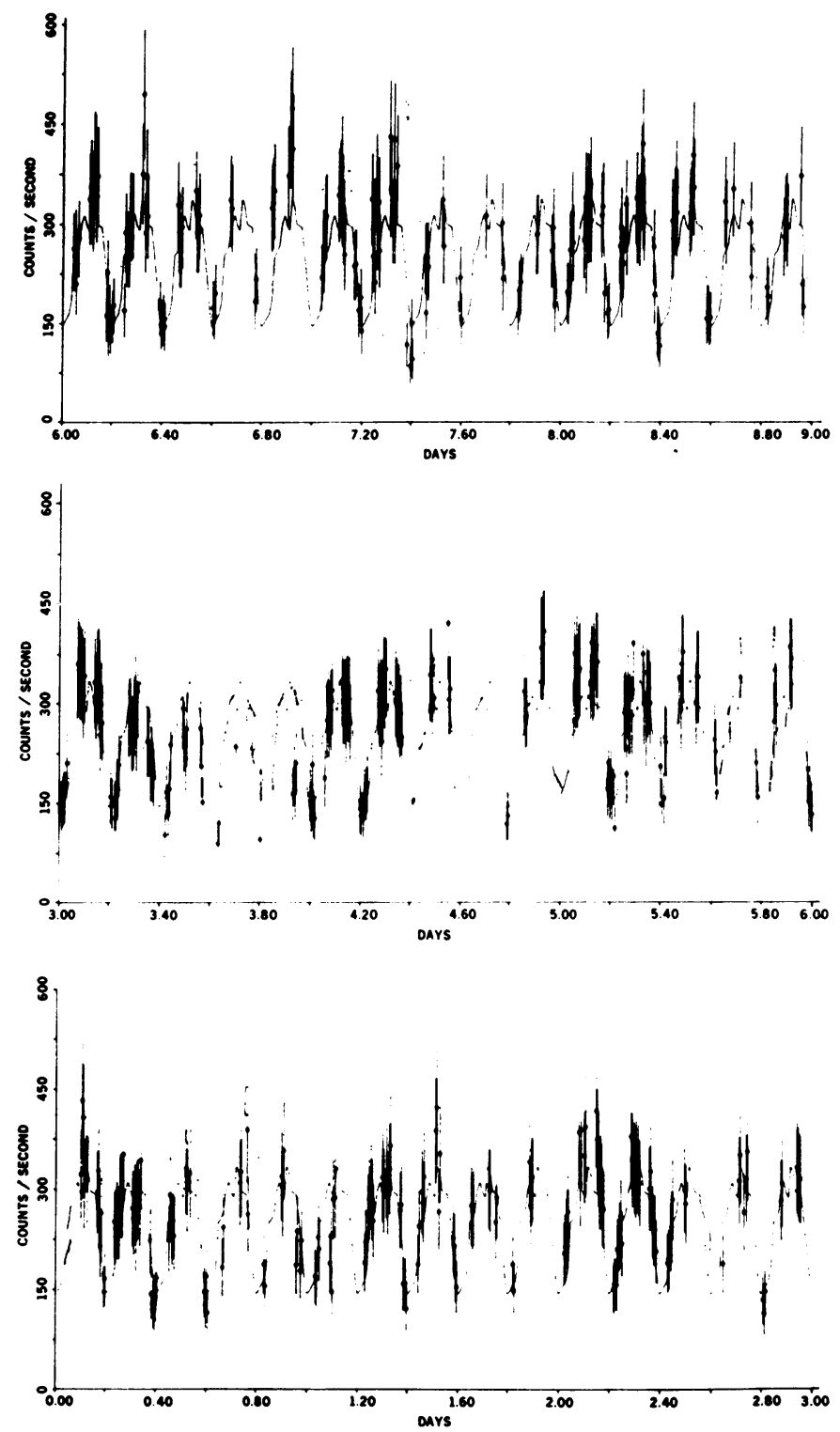

Fig. 18. Observations of Cygnus X-3 from 1972, May 8 to May 17 are plotted as a function of time. The intensities are given in $\mathrm{ct} \mathrm{s}^{-1}$ and have been corrected for aspect; error bars including both statistical error and systematic error due to aspect correction are shown. The time scale is in days with day $0.0=$ May 8.0 (UT). Also shown is the average light curve obtained by folding all the data modulo $4.80 \mathrm{~h}$, and finding the average intensity every $0.24 \mathrm{~h}$. 
of four and persisting for possibly months have been seen. Although the X-ray intensity varies on times short with respect to $4.8 \mathrm{~h}$, no significant fluctuations have been seen in times on the order of tenths of seconds.

The radio counterpart of Cygnus X-3 is extremely variable, ranging from 20 milliflux units to 20 flux units in several days (Braes et al., 1972; Gregory et al., 1972; Hjellming et al., 1972). This includes the first giant radio flare of September 1972. The X-ray source was very active on the day before this radio flare was first observed; intensities a factor of two greater than previously seen were reported. The X-ray intensity observed from Uhuru at the time of the flare is shown in Figure 19. The $\mathrm{X}$-ray spectrum also appeared hotter for the high intensity points.

Becklin et al. (1973) have reported coordinated X-ray and infrared observations of Cyg X-3. The X-ray and infrared fluxes vary in phase with a 4.8-h period, although there were observations when no periodic variations in the infrared were seen. In addition, there is unpredictable large amplitude flaring in the infrared flux. The agreement in 4.8-h period establishes the association of the $\mathrm{X}$-ray with the infrared source and through the positional coincidence $\left(2^{\prime \prime}\right)$ between the infrared and radio source, of the X-ray and radio source. However, on a later occasion, (October, 1973) Becklin and Neugebauer (1974) failed to detect the 4.8-h period in the infrared even though the source was present at close to its previously observed intensity.

The fact that Cyg X-3 displays a similar in-phase 4.8-h periodicity at wavelengths ranging from the $\mathrm{X}$-rays to the infrared at least some of the time, strongly suggests that it is an eclipsing binary system. Additional evidence in favor of this explanation is (a) the relative flatness of the infrared maxima, (b) the fact that the relative flux differences between maximum and minimum appear to be constant at different epochs despite changes in both the mean infrared flux and mean $\mathrm{X}$-ray flux, and (c) the fact that the flare activity is apparently uncorrelated with the phase of the 4.8-h variation.

In a binary system with total mass $\mathfrak{M}$ and period $4.8 \mathrm{~h}$, the separation between the stellar centers is $1.4\left(\mathfrak{M} / \mathfrak{M}_{\odot}\right)^{1 / 3} R_{\odot}$. From the shape of the eclipse curve it can be concluded that the system is a contact, or near-contact, binary with each component on the order of one solar radius. Taking $10 \mathrm{kpc}$ as the lower limit for the distance to the source (Braes et al., 1973) and taking $6 \times 10^{-28} \mathrm{~W} \mathrm{~m}^{-2} \mathrm{~Hz}^{-1}$ as the dereddened $2.2 \mu$ flux density of the source, Becklin et al. (1973) obtained a $2.2 \mu$ surface brightness temperature.

$$
T_{B} \sim 6 \times 10^{6}\left(\frac{\mathfrak{M}}{\mathfrak{M}_{\odot}}\right)^{-2 / 3}\left(\frac{R}{R_{\odot}}\right)^{-2} \mathrm{~K} .
$$

For any reasonable total mass of the Cyg X-3 binary system, the object seen at $2.2 \mu$ has a surface temperature higher than that of the photosphere of any normal star. The high estimated value for the infrared surface brightness, and the similarity of the $\mathrm{X}$-ray and infrared eclipse curve, suggests that both the X-ray and the infrared fluxes originate from the same hot object.

The phenomenological picture of Cyg X-3 as a contact system in which each com- 


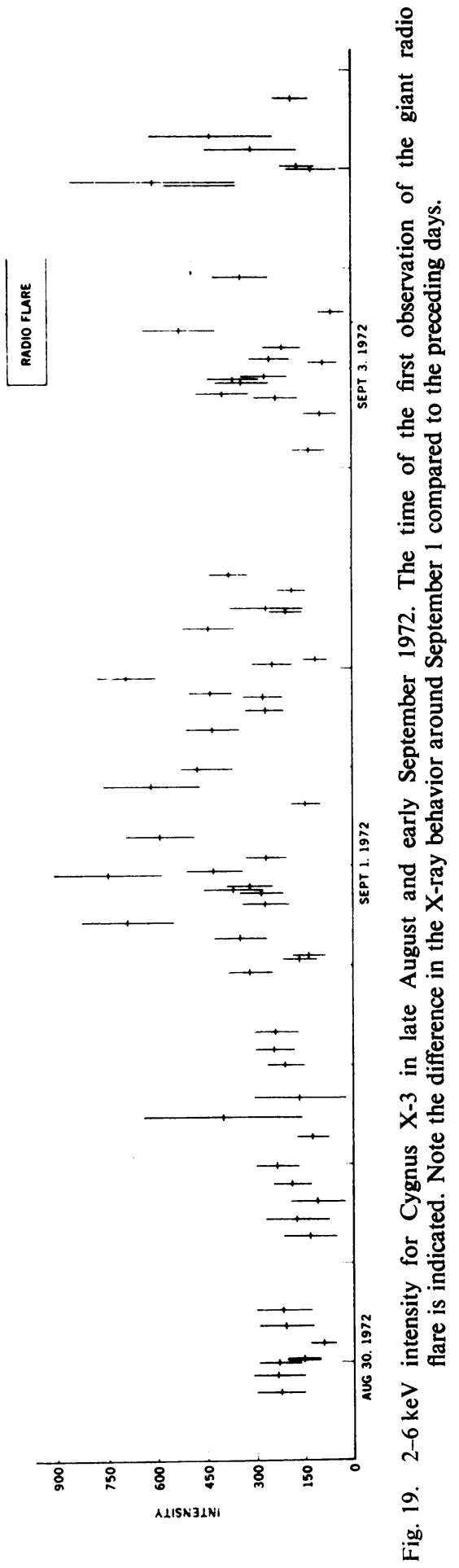


ponent has a radius $\sim 1$ solar radius would appear to indicate that it is a completely different kind of object from Her X-1 and Cyg X-1 which are compact objects. However, Basko et al. (1973) have shown how the X-ray light curve in Cyg X-3 can be explained by the reflection of $\mathrm{X}$-rays by the atmosphere of the normal companion in a close binary. If Cyg X-3 is an X-ray pulsar in which the beam never crosses the Earth but continuously strikes the surface of the normal counterpart, then the reflected X-rays would be the only flux detected at Earth, with a $4.8 \mathrm{~h}$ period smooth, $\mathrm{X}$-ray light curve. In this model, the $\mathrm{X}$-ray flux should be $\lesssim 50 \%$ polarized, and a jump at the K-edges of iron might be detectable in the spectrum. No attempt has been made to incorporate the flaring in the radio and infrared into a binary picture. The connection of magnetic field lines in an accretion disk (Sunyaev, 1973) in a manner analogous to solar flares could lead to the acceleration of electrons to sufficient energies for non-thermal synchrotron emission to occur (Blumenthal and Tucker, 1972). An alternative, simpler phenomenological picture just places the compact object within the atmosphere of the primary. The standard Roche model and accretion disk is then not necessarily relevant. Any short time scale variations are averaged out by the gas surrounding the source. The 4.8-h variation is then caused by Thompson scattering by the hot gas close to the system; the non-variable 2.5-3 keV low energy cutoff is caused by photoelectric absorption by the cooler gas further out. (See e.g., Davidsen and Ostriker (1974) for a model invoking a red dwarf - white dwarf binary, although they predict a periodic low energy cutoff.)

\subsection{SCORPIUS $X-1$}

Sco $\mathrm{X}-1$ is the brightest source in the sky in the $1-10 \mathrm{keV}$ range. It was the first $\mathrm{X}$-ray source discovered and has been extensively studied, yet its nature is still a mystery. The tendency is to assume that it is a close binary source, but the evidence in support of this is only circumstantial.

The $\mathrm{X}$-ray spectrum in the $\mathrm{keV}$ region is best fit by an exponential with an $e$-folding energy that varies with time but is on the average about $4 \mathrm{keV}$ (Oda and Matsuoka, 1970). There is no evidence for periodic or even random pulsations of more than a few percent on a time scale ranging from a few milliseconds to about 1 minute (Boldt et al., 1971). However variations on the order of $50 \%$ or more have been observed on time scales of hours and days (Tananbaum, 1973). Above about $30 \mathrm{keV}$ the spectrum departs from this behavior and exhibits a 'non-thermal tail' which is highly variable (Peterson, 1973). The optical and infrared continuum is consistent with an extrapolation of a flat spectrum from X-ray energies, with absorption becoming important in the infrared (Neugebauer et al., 1969). Optical distance indicators such as reddening, polarization, or $\mathrm{CaK}$ absorption indicate a distance greater than 200 pc.

Optically, Sco X-1 varies between 12-13 mag. The intensity varies by a few percent on a time scale of minutes and flares by a factor of two on a time scale of hours. There appear to be both active and passive states. Correlated optical/X-ray studies show that when the source is quiescent optically, the X-ray emission is also quiescent, 
and the variations in optical and X-ray radiation are weakly coupled, if at all. When the source is active optically, it is also active in X-rays, and the variations are strongly correlated, as shown in Figure 20 (Tananbaum, 1973; Price et al., 1971). This suggests that some of the optical emission is generated in the hot X-ray emitting region, which dominates when the source is active, with the remainder originating in a physically distinct cooler region which dominates when the source is quiescent. The spectrum also shows a number of high excitation emission lines which must originate in a separate cooler region. There is no evidence for a periodic component in the optical intensity or for a Doppler shift of the spectral lines, although the spectral lines do vary in both intensity and position.
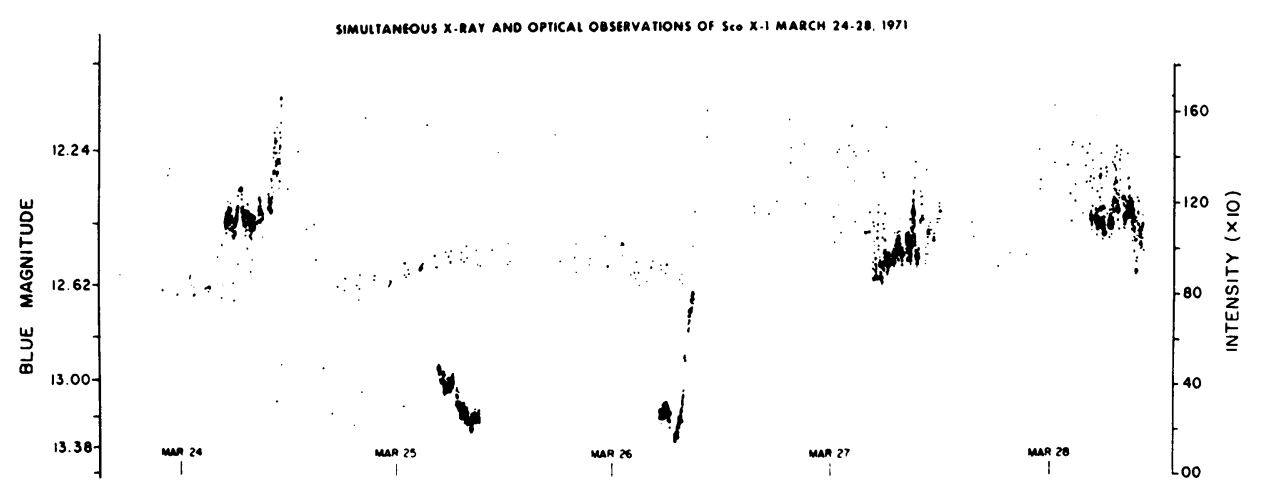

Fig. 20. Simultaneous X-ray (low density data points) and optical (high density) observations of Sco X. The X-ray and optical data exhibit correlated periods of quiet and active states.

Several unsuccessful or inconclusive attempts have been made to observe X-ray emission lines from sulfur ions, which should make a contribution of the order of $1 \%$ to the total intensity in the rage $1-10 \mathrm{keV}$ if the plasma is optically thin. The upper limits on observed lines imply that the electron scattering optical depth is about 10 for the Sco X-1 source (Stockman et al., 1973; Felten et al., 1972). These results, together with the total observed luminosity, implies that the characteristic size of Sco X-1 is about $10^{9} \mathrm{~cm}$ or less and the density about $10^{16} \mathrm{~cm}^{-3}$ or greater.

Optical data obtained at the time of its identification in 1966 was similar to that obtained from old novae, leading to the belief that the $\mathrm{X}$-ray emission originated near a white dwarf in a close binary system, in analogy to the current ideas concerning old novae. This idea was strengthened by the infrared observations of Neugebauer et al. (1969) described above. If it were assumed that the optical and X-ray continuum originated in a common volume, the infrared results required an emission region $\sim 10^{4}-10^{5} \mathrm{~km}$ in size; i.e., a size appropriate to a white dwarf. However, the data in Figure 21, which are a portion of these in Figure 20, show in detail the transition from a quiet to an active state. The optical continuum comes on about one hour before the X-ray. This requires a structured region, as had been described earlier by Shklovski (1967) and is not compatible with the common volume for both the X-ray 


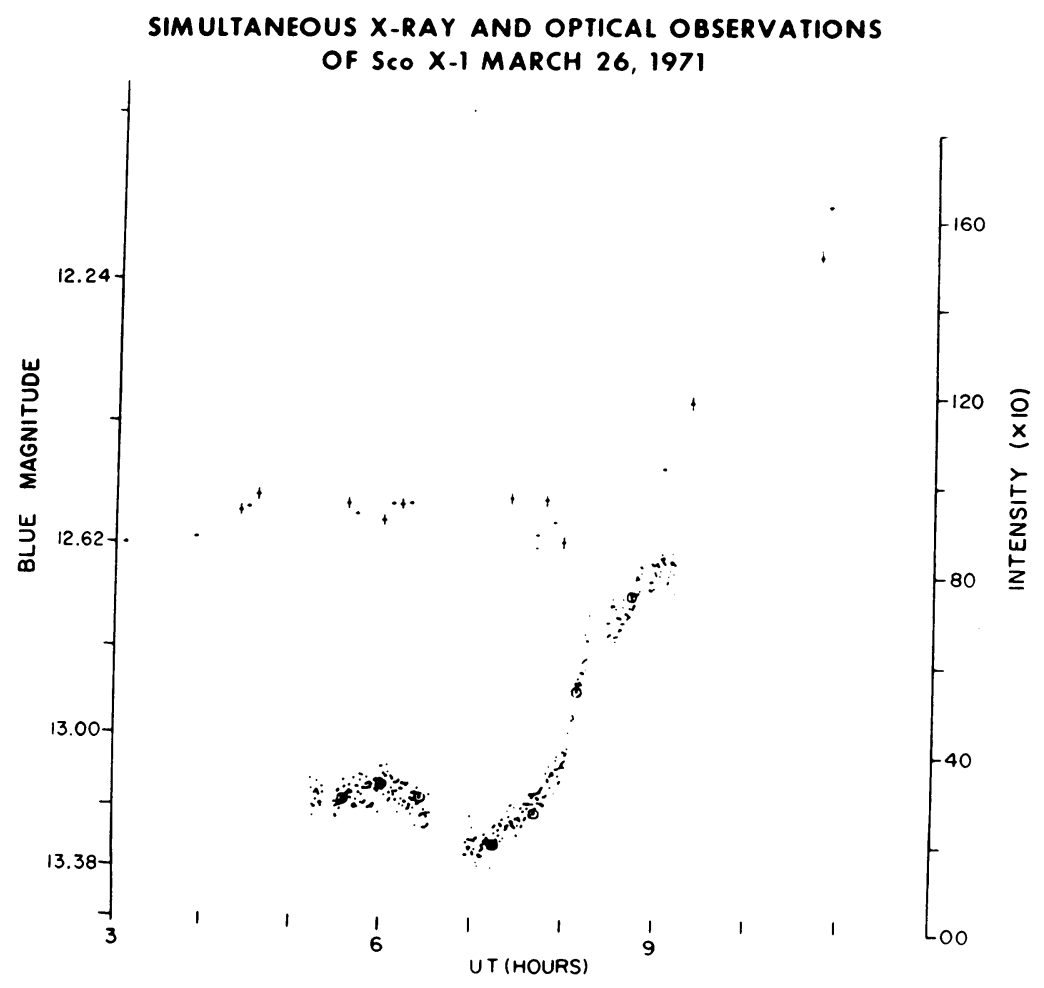

Fig. 21. Detail of X-ray-optical simultaneous transition of 26 March 1971. Optical transition occurs significantly earlier than the X-ray transition.

and optical emissions. Thus, the infrared (and optical) may be produced in a region of white dwarf size, but the X-rays may be produced in a much smaller, inner region.

If Sco X-1 is an accreting close binary, then the orbit must have a large inclination to the line of sight. This would explain the absence of eclipses, of Doppler variation in the optical emission lines, and might explain the absence of a large and variable low-energy X-ray cutoff. The primary must be a low mass object because it is not bright in the optical or infrared. Basko and Sunyaev (1973) have proposed a model in which the primary is a star with $1 \mathfrak{M}_{\odot}$ and the secondary is a black hole.

\subsection{Cygnus X-2}

Cygnus X-2 is believed to be similar to Sco X-1 except there is not nearly so much information available concerning it. The $1-10 \mathrm{keV}$ spectrum is best fitted by an exponential with $k T \simeq 4 \mathrm{keV}$. The flux varies by as much as $25 \%$ in a second, on occasion. Cyg X-2 has a strong, variable high energy component which can account for as much as $15 \%$ of the total intensity (Matteson, 1971). An optical identification was proposed in 1967 (Giacconi et al., 1967) based on the discovery of a star with characteristics similar to Sco X-1 within the area of uncertainty of the X-ray source. The X-ray position has since been refined, still including the candidate star and 
excluding a suggested non-variable radio source. However, simultaneous X-ray and optical variations have not yet been reported, making the identification only likely and not yet positive. In both the optical and X-ray, variations in intensity of order a factor of 2 are observed within a day; however, the 2 states characteristic of Sco X-1 have not yet been reported for Cyg X-2, possibly because of much less available data.

In the optical, Cyg X-2 is actually very different from Sco X-1, although they have some common features, such as variable intensity and an ultraviolet excess. In Cyg $\mathrm{X}-2$, the spectral lines show large changes in radial velocity which were at first thought to be evidence of binary motion; however, Kraft and Demoulin (1967) showed that these variations, which are of several hundred $\mathrm{km} \mathrm{s}^{-1}$ were not periodic. Furthermore, they found that the bulk of the optical emission could be accounted for by the presence of a G-type sub-dwarf based on the absorption line spectrum. They estimated a distance of $500-700 \mathrm{pc}$, implying an X-ray luminosity of order $10^{36} \mathrm{erg} \mathrm{s}^{-1}$.

With this distance, the optical emission would then be $10^{34} \mathrm{erg} \mathrm{s}^{-1}$ and is dominated by the F-type star. The optical emission from the $\mathrm{X}$-ray region could then be $10^{33} \mathrm{erg} \mathrm{s}^{-1}$ or $10^{-3}$ of the X-ray emission as in Sco X-1. Any radio emission from Cygnus X-2 might be considerably less than for Sco X-1, making it difficult to detect. In any case, the presence of a G-type sub-dwarf along with an X-ray source is evidence for the presence of two stars in the system, if the identification is correct.

Several other objects appear to have similar X-ray properties to Sco X-1 and Cygnus X-2. They include GX $17+2$ (2U $1813-14)$, GX $3+1$ (2U $1744-26)$, GX $5-1$ (2U $1757-25)$, GX $9+1$ (2U $1758-20)$, and GX 349+2 (2U $1702-36)$, all of which have intensity variations of order factors of 2 within a day and exponential spectra with temperatures varying from 50 to $150 \times 10^{6} \mathrm{~K}$. These five additional sources all have about a $2 \mathrm{keV}$ cutoff suggesting distances of the order of $10 \mathrm{kpc}$ (if the cutoffs are caused by interstellar absorption) and thus X-ray luminosities of the order of $10^{38} \mathrm{erg} \mathrm{s}^{-1}$.

\subsection{OTHER BINARIES}

We return now to the remainder of the X-ray sources which have been found to be binaries.

\subsection{1. $3 U 1700-37$}

The source $3 \mathrm{U} 1700-37$ was discovered by Uhuru and was found to have a period of 3.412 days. The source is on for 2.3 days and off for 1.1 days, the longest occultation of any of the X-ray binaries. There is some indication of a secondary minimum at phase 0.5 . The binary nature of the X-ray emission is shown in Figure 22. The intensity is very variable on time scales of hours down to tenths of a second. The fluctuations, however, are not periodic. The X-ray spectrum is flat with a variable low energy cutoff which is comparable to or greater than that of the other eclipsing $\mathrm{X}$-ray binaries.

The optical identification of 3U 1700-37 with the star HD 153919 was suggested by Jones et al. (1973) and confirmed by a large number of observers. The star is an 
early supergiant, a $6.6 \mathrm{mag}$. $07 \mathrm{f}$ at a distance of $1.7 \mathrm{kpc}$. It displays a double-peaked light curve with an amplitude of about $0 .{ }^{m} 1$ and a period of 3.4 days; one of the minima coincides with the X-ray eclipse. Spectroscopic observations by Hutchings et al. (1973) determined radial velocity variations of amplitude $29 \pm 6 \mathrm{~km} \mathrm{~s}^{-1}$; similar results were obtained by Hensberge et al. (1973) and Wolff and Morrison (1973). Strong emission lines are present, with emission and absorption lines showing different radial velocities indicating an expanding atmosphere.

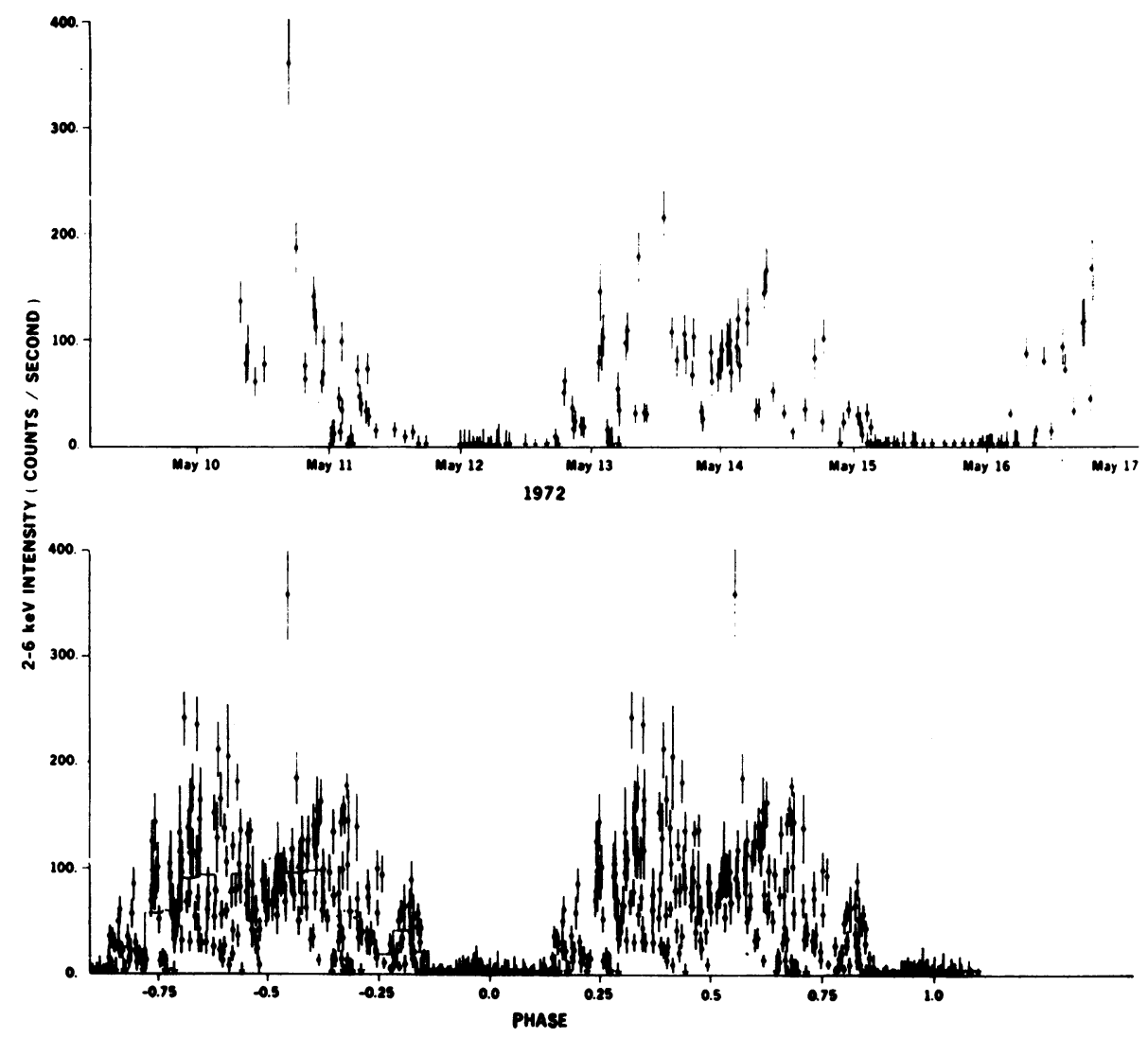

Fig. 22. The 2-6 keV intensity of $2 U 1700-37$ corrected for elevation in the field of view and shown with $\pm 1 \sigma$ error bars. The upper portion shows seven days of data from May 1972. Plotted below are observations obtained between December 1970 and May 1972 folded with the 3.412-day period.

If the mass of the primary is that of a typical $07 \mathrm{f}$ star, at least $35 \mathfrak{M} \lambda_{\odot}$, then the minimum mass of the $\mathrm{X}$-ray source is $1.4 \mathfrak{M}_{\odot}$. Although the system as a whole bears a strong resemblance to Cyg X-1, the minimum mass and the present $\mathrm{X}$-ray data cannot determine whether the $X$-ray source is a neutron star, a degenerate dwarf, or a black hole. 


\subsection{2. $3 U 0900-40$}

$3 \mathrm{U} 0900-40$ is a highly variable source. It was found to be an eclipsing binary by Ulmer et al. (1972) and Forman et al. (1973). The period is 8.95 days with an eclipse duration of 1.85 days. Its light curve (and variability) is shown by the folded Uhuru data in Figure 23. The $\mathrm{X}$-ray intensity is found to be variable on time scales of seconds to days; on one occasion the intensity increased by a factor of 30 in $2 \mathrm{~h}$. But no periodic variations have been seen.

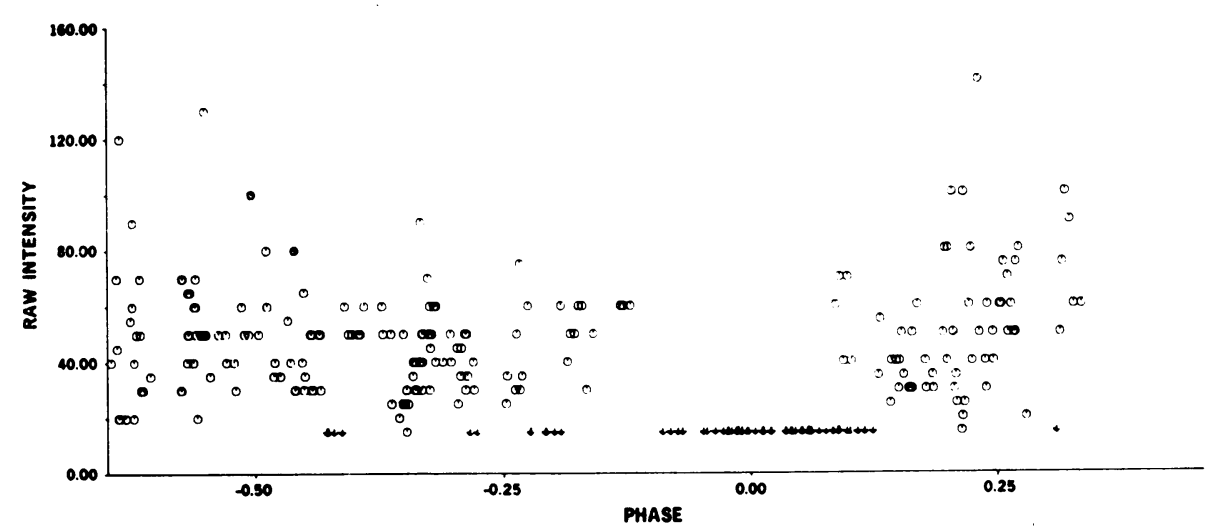

Fig. 23. The observed 2-6 keV X-ray counting rates (uncorrected for aspect) for $2 U 0900-40$ from May to July 1972 folded modulo 8.95 days. The absence of any high sightings around phase 0.0 shows the eclipsing behavior of the source.

The X-ray source has been identified with HD 77581, a 6.9 mag. B0.5Ib star at $1.3 \mathrm{kpc}$. Both spectroscopic and photoelectric observations show agreement in period and phase between the optical and X-rays (Hiltner et al., 1972; Hutchings, 1972; Jones and Liller, 1973; and others). The light curve is double peaked with an amplitude of 0.1 mag., similar to $3 \mathrm{U} 1700-37$, but exhibits significant variability. As with $3 \mathrm{U} 1700-37$, if normal mass is assumed for the primary, then spectroscopic observations imply a mass of about $1.7 \mathfrak{M}_{\odot}$ for the X-ray source (see e.g., Hutchings et al., 1973).

\subsubsection{Circinus $X-1$ ( $3 U$ 1516-56)}

The X-ray behavior of Cir X-1 is very similar to that of Cyg X-1. The source has been observed to vary significantly on all time scales observed down to $0.1 \mathrm{~s}$. Pulse trains have been observed, but no periodicities have been found to persist as with Cyg X-1 (Schreier et al., 1971; Margon et al., 1971). Different average intensity levels have been seen, with different average spectra. The variability from day to day appears greater than that for Cyg X-1. The data suggests eclipsing behavior, although many low points are seen outside of eclipses; it is likely that the binary period is longer than 12 days (Jones et al., 1974). 
The accumulated observations taken over a period of two years from Uhuru shown in Figure 24 reveal several instances (February 1971, November 1971, and April 1972) of about a month duration when the source was more intense by about a factor of three than other times. The spectrum of the radiation during the times of high intensity contains a soft component which can account for the bulk of the intensity difference. This behavior is very similar, in time scale, relative intensity and spectrum, to the two states seen in Cyg X-1 (see Figure 5).

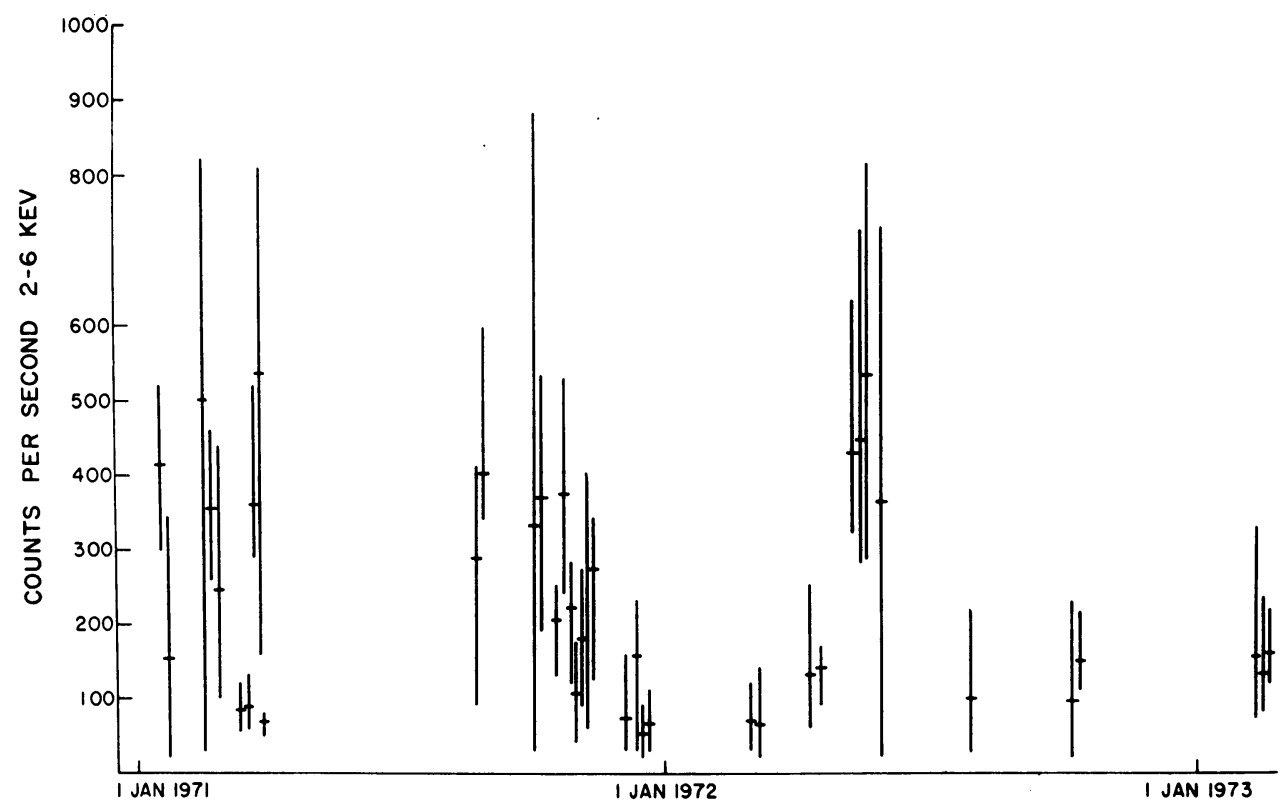

Fig. 24. The intensity of Circinus X-1 as measured over a two-year period by Uhuru. Vertical extensions of data points are not uncertainties but are the observed variation in intensity on the day of observation.

No star brighter than 14th magnitude is present in the $0.0002 \mathrm{deg}^{2}$ error box for the source. Thus, although the X-ray characteristics resemble closely those of Cyg X-1, the companion star appears quite dim. This could be due to obscuration or merely to a lower mass for the companion.

\subsection{4. $S M C X-1(3 U 0115-73)$}

SMC X-1 is the only optically identified X-ray source in an external galaxy. The source exhibits a 3.893-day period, with a X-ray eclipse lasting 0.6 days as shown in Figure 25 (Schreier et al., 1972b). Significant intensity fluctuations have been observed on time scales of hours and perhaps minutes. In addition, extended lows have been observed. The source is too weak to determine whether fluctuations exist on short time scales. However, no regular short period pulsations have been observed with an upper limit of $10 \%$ of the power. The distance to the SMC of $61 \mathrm{kpc}$ implies a 2-6 keV 
luminosity of $10^{38} \mathrm{erg} \mathrm{s}^{-1}$. The observation of flux at $40 \mathrm{keV}$ (Prince et al., 1971) extends the integrated X-ray luminosity to over $10^{39} \mathrm{erg} \mathrm{s}^{-1}$, making it probably the strongest stellar X-ray source. This luminosity is comparable to or greater than the usual Eddington limit for accreting sources.
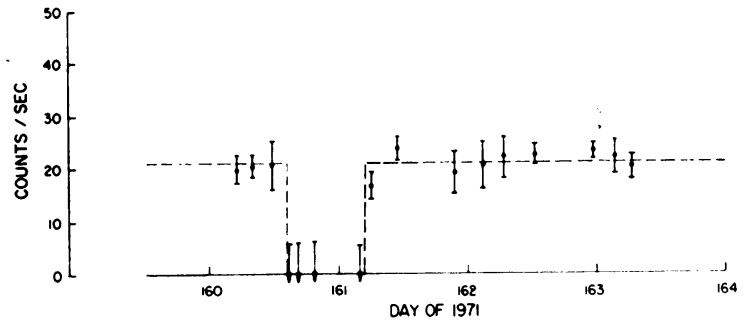

$\underbrace{50}_{0}{ }^{50}$

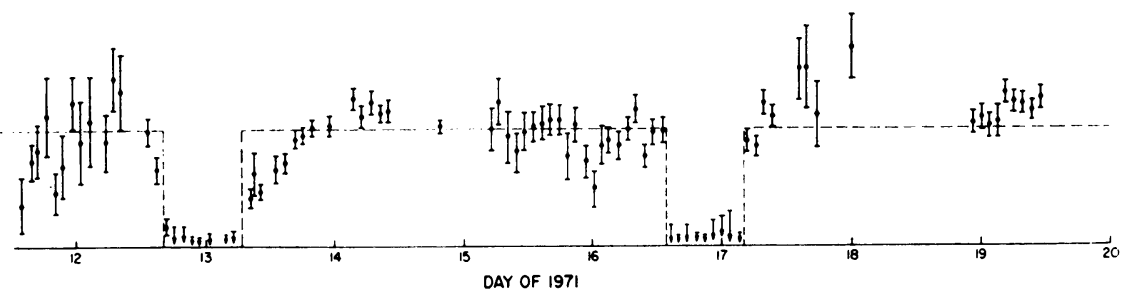

Fig. 25. The intensity of SMC X-1 in observed ct $\mathrm{s}^{-1}$ during eight days in January and three days in June 1971. Data have been corrected for elevation in the field of view; $1-\sigma$ error bars include both statistical and elevation correction errors.

The star Sanduleak 160 was proposed as the optical counterpart for SMC XI by Webster et al. (1972) and confirmed by Liller (1973). It is a 13.2 mag. B0I supergiant. The star exhibits a double-peaked light curve of the correct period, with amplitude variation of about $0.1 \mathrm{mag}$. The absolute magnitude of the star is calculated as -6.0 . This is what is expected for this spectral type star, indicating that the presence of a strong X-ray source has not significantly altered the spectral appearance of the star. This is relevant in countering arguments that the spectral type of a binary companion can not be used in determining mass due to the effect of the X-ray source (i.e., for Cyg X-1). It should be noted that the ratio of X-ray to optical emission from SMC X-1 is greater than for Cyg X-1.

Spectroscopic measurements by Osmer and Hiltner (1974) suggest that the mass of the X-ray source is between 1 and $4 \mathfrak{M}_{\odot}$.

\subsection{THE CRAB NEBULA AND NP 0532}

Even though this paper began with a disclaimer that supernova remnants would not be considered and it is established that the Crab pulsar, NP 0532 is a single star to a high degree of precision, a curious aspect of the X-ray behavior of the Crab has recently been found in the Uhuru data which may have a bearing on the processes comprising the emission from the binary X-ray sources (Forman et al., 1974). 
There has been a continuing search among the $\mathrm{X}$-ray sources for short time variability as was found in Cyg X-1. Uhuru source observations were typically 0.096-s consecutive samples of 2 or $20 \mathrm{~s}$ total duration. If one does a $\chi^{2}$ fit to the count rate observed in the individual samples of a single observation on the assumption that the source intensity is constant, a value of $\chi^{2} \mathrm{deg}^{-1}$ of freedom of about four, typically.

The Crab was included in this study since it was not expected to show any such effect. The poor time resolution reduces the contribution to excess variability of the pulsar to about $1 \%$, which is too small to be detected. However, on several occasions, for periods of about one day, the $\chi^{2} \mathrm{deg}^{-1}$ of freedom was considerably in excess of unity. One observation taken is shown in Figure 26. On these occasions the X-ray power which is variable must be $\sim 10$ to $20 \%$ of the total Crab power. The power from the pulsar is only about $10 \%$ in this energy range. On most occasions, the Crab shows no such effect.

The temptation is to associate this variability with variations in the pulsar power, as is seen in radio. However, it is possible that a completely different source of X-rays is present which is varying erratically for the same reason that other galactic X-ray sources vary erratically on this short time scale.

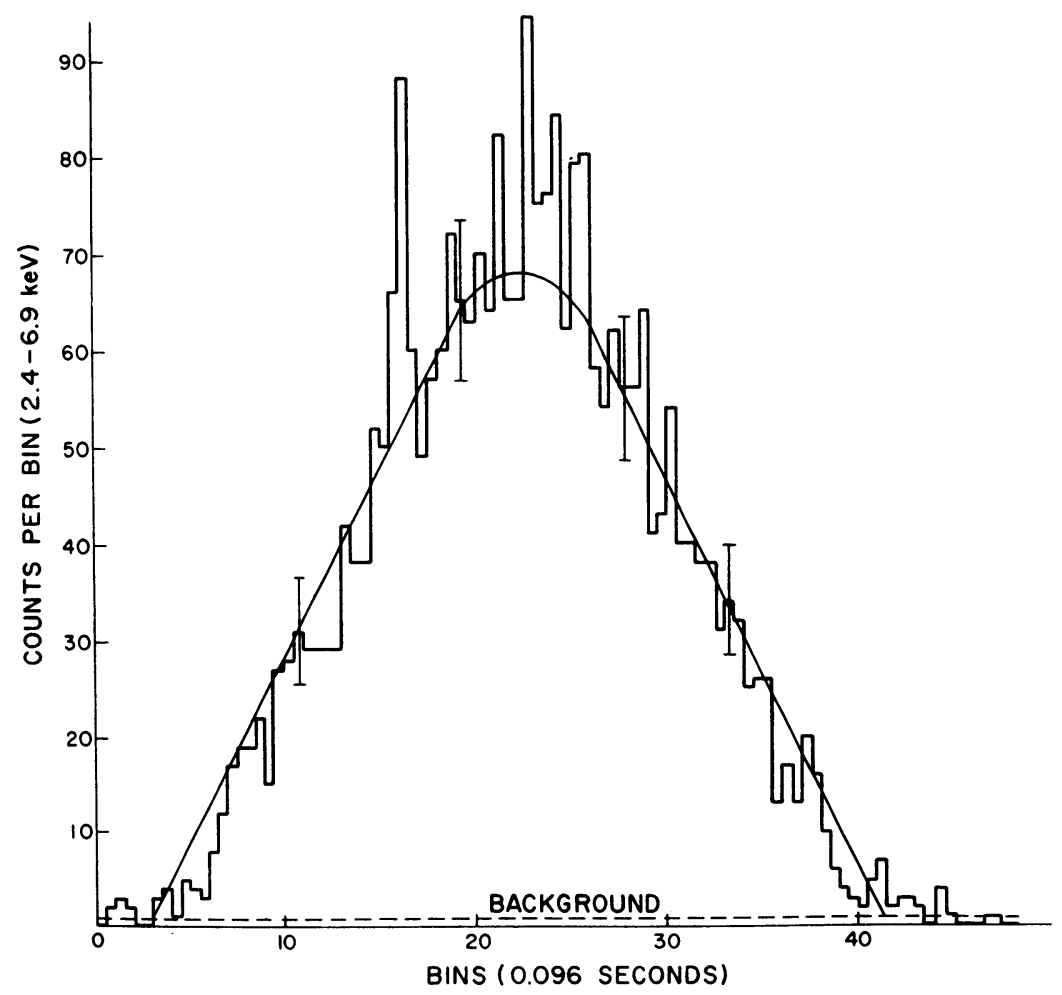

Fig. 26. Single transit of the Crab Nebula by Uhuru showing excess fluctuations. The single large spike to left of peak is about $4 \sigma$ off the best fit to the data. 


\section{A Standard Model for the X-Ray Sources - Close Binary Systems and Accretion}

The presence of a compact object in a close binary system presents a natural and efficient means of producing X-rays. As matter falls onto a compact object, gravitational potential energy is released, heating the matter and producing radiation. The efficiency of this process is such that modest accretion rates can produce the luminosities and temperatures characteristic of galactic X-ray sources. Thus, it is not wholly surprising that theoretical models along these lines were suggested quite early, as has been already discussed. We suggest here that in fact the observations favor this view; furthermore, we suggest that the observations favor a neutron star or a black hole as the actual X-ray source. There is a variety of evidence that leads to this conclusion. However, it is implicit in these arguments that the $\mathrm{X}$-ray sources comprise a single kind of stellar system. This allows the drawing together of the evidence on the individual sources into the construction of a single model. There is some justification for this view. For one, there is a category of X-ray sources associated with a very specific companion; namely, a B0 supergiant. This specificity implies that the X-ray source itself is also a certain kind of stellar object, which we argue is either a neutron star or a black hole. From the point of view of making X-rays by accretion, a neutron star and a black hole are very similar since their sizes are comparable. To invoke white dwarfs, however, requires qualitatively different conditions since their size is $10^{3}$ times greater. One consequence of this larger size is that the mass accretion rate must be $10^{3}$ times greater than is the case for a neutron star or a black hole.

The other category of X-ray sources is associated with more common stars and the evidence is not so compelling that they comprise so narrow a class of stellar systems. There is at least one cross link between these two categories of X-ray sources and that is the two pulsing X-ray sources. Cen X-3 is an example of an X-ray source associated with a B0 supergiant whereas $\mathrm{Her} X-1$ is associated with a low mass, halo or intermediate population star.

Another argument for a 'standard' model is the rarity of the X-ray sources. The presently observed sources comprise only 1 in $10^{9}$ of all stars in the Galaxy, which is a very small number. The probability that nature has found a variety of means to make X-ray sources with approximately the observed space density must be exceedingly remote.

The direct evidence for the standard model is as follows. A number of the X-ray sources are positively confirmed as being in binary systems with periods varying from 0.2 days to 12 days. Considering the probability of seeing the binary character, the data are consistent with all the $\mathrm{X}$-ray sources being binaries. There is direct evidence for large mass transfer rates in many of these systems. The 'dips' in Her X-1 are absorption features and are directly interpretable as due to a gas stream. In both Cen X-3 and Her X-1, the pulse period changes and possibly orbital period changes are consistent. In fact, in these two objects, as will be discussed below, the observed pulse period change is more compatible with a rotating object of neutron star size 
than one of white dwarf size. A more direct argument that these two objects involve neutron stars is simply that it has not been possible to find a means of making a white dwarf rotate or vibrate stably with periods as short as a second (cf. Ostriker, 1971).

Additional direct evidence for the nature of the X-ray source is in the case of Cygnus X-1 where the high mass requires a black hole.

In the remainder of this section, we describe what may be the inner structure of the X-ray emitting systems.

\subsection{THE BINARY SYSTEM}

A standard means of describing binary star systems is via the Roche model (see e.g. Kopal, 1959). One uses a coordinate system rotating with the binary, assumes circular orbits and co-rotating stars, and defines the equipotentials of the system as in Figure 27. Close to each star, the surfaces are spherical; they then gradually increase in size and deform until the surfaces around each star touch at $L_{1}$, the inner Lagrangian point. This first common equipotential is called the Roche lobe or critical surface. If one star fills its Roche lobe, then matter can pass through $L_{1}$ toward the

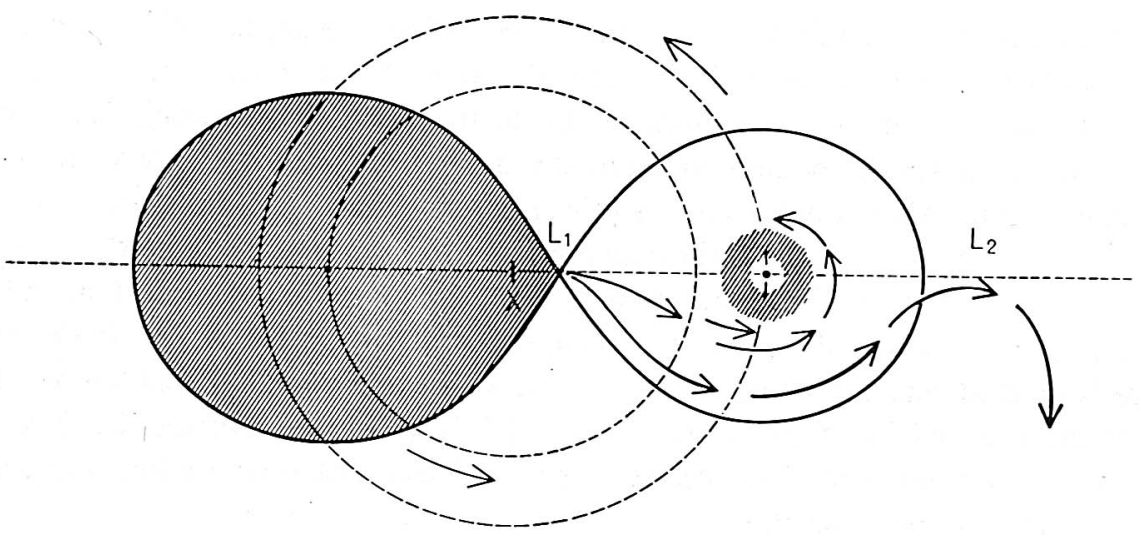

Fig. 27. Roche equipotential surfaces (lobes) are shown by the darkest line. One star (cross-hatching) is assumed to fill its lobe and spill material through the inner Lagrange point $\left(L_{1}\right)$. The flow of the material is shown by the arrows. Some may end up on the companion star and some may leave the system through the outer Lagrange point $\left(L_{2}\right)$.

other star. In this case, the angular momentum of the accreted matter causes a disk to form around the secondary. Alternatively, mass transfer can also occur if one star has a stellar wind from which matter can accrete onto the secondary. This stellar wind can either be of a conventional type from a massive star, or can possibly be driven by the companion X-ray source which heats the outer layers of the star. Either of these means of mass transfer may be present in the observed binary $X$-ray sources, and it is possible that both of them are relevant to some sources.

The stellar wind models have been discussed in the literature; either of the types can yield sufficient mass to power an X-ray source (Davidson and Ostriker, 1973). 
There is also an argument that the $\mathrm{X}$-ray induced wind will produce $\mathrm{X}$-ray luminosities close to the Eddington limit, $\sim 10^{38}\left(\mathfrak{M}_{x} / \mathfrak{M}_{\odot}\right)$ erg s${ }^{-1}$ (Arons, 1973; Basko and Sunyaev, 1973). There are two features of the stellar wind model which seem particularly relevant. The first involves the fact that a very strong stellar wind may 'bury' the $\mathrm{X}$-ray source, effectively absorbing the $\mathrm{X}$-rays and re-emitting the radiation at longer wavelengths (see e.g. Pringle, 1973). This can be used to explain the extended lows of some of the sources, especially an irregular one like that of Cen X-3. The repeating but non-periodic extended lows would then be tied to the variability of the stellar wind of the supergiant primary. (The 35-day cycle of Her X-1 can as well be explained by a precession-timed 'magnetic gate', allowing accretion only when the magnetic pole is within the accretion disk.) The second feature of a stellar wind model is the fact that radial mass loss will increase the binary period. It is interesting to note that both mass transfer (from the more massive to the less massive component) and mass loss from the system through $L_{2}$ in a Roche model will tend to decrease the period (see e.g. Kruszewski, 1966). Thus, in Cen X-3 where the period is seen to change in both directions, the radial stellar wind slow-down effect may at times predominate over the speed-up effects.

\subsection{THE ACCRETION DISK AND COMPACT OBJECT}

The relevance of accretion disk models for compact sources has been discussed in some detail (see e.g. Prendergast and Burbidge, 1968; Schwartzman, 1971; Pringle and Rees, 1972; Shakura and Sunyaev, 1973). An accretion disk is required in the normal Roche picture of binary mass transfer. The accreting matter will have too much angular momentum to fall radially onto the secondary; it will instead form a disk and the material will spiral inward as the angular momentum is dissipated. It is also important to note that a black hole model must contain an accretion disk to emit X-rays. The in-fall time of matter accreting radially into a black hole is probably too short to allow sufficient radiation, whereas matter accreting radially onto a neutron star can deposit its energy on the surface which then radiates as a black body.

The properties of the accretion disk, and of the radiation from it, are dependent upon the detailed assumptions about its structure, its viscosity, etc. However, reasonable models have been constructed from which can be estimated the X-ray emissivity (see e.g. Pringle and Rees, 1972; Shakura and Sunyaev, 1973). The X-rays can be due to bremsstrahlung or black body radiation depending on the density of a gas at any given point. Integrating the radiation over the entire disk with its radially dependent temperature can then produce a non-thermal power law spectrum consistent with observations. But, in fact, the main variable in the model is probably the inner boundary condition; namely, the nature of the compact object present at the center. If the central object is a black hole, then the disk can extend inward to the closest stable orbit, corresponding to a period of about $10^{-3}\left(\mathfrak{M} / \mathfrak{M}_{\odot}\right)$ seconds or a factor of a few less for a Kerr black hole. The effective in-fall time is some 100 times this period, leading to a characteristic time scale for pulsations or fluctuations between $10^{-3}$ and $10^{-1} \mathrm{~s}$. The radiation is also highly dependent on the mass accretion rate. 
Pringle and Rees (1972) point out that significant flaring is expected if the mass flux is either so large that the Eddington limit is exceeded, or so small that energy cannot be radiated efficiently.

If the central object is a neutron star, then the accretion disk is probably not the dominant influence on the radiation. The disk either extends inward to the surface of the star, in which case both the disk and the star contribute to the emission, or it extends down to the Alfven surface. This is the point at which the stellar magnetic field dominates the accretion flow; it can be considered as the radius at which the magnetic field energy density becomes comparable to the kinetic energy of the accreting matter. The in-falling matter then follows the field lines and thus must corotate and flow toward the magnetic poles. The enforced co-rotation exerts a torque on the star. The sign depends on the relative sense of the orbital and spin rotations, and the time scales are on the order of $10^{4}$ or $10^{5} \mathrm{yr}$.

An important consideration at this point is the effect of centrifugal forces (see e.g. Lamb et al., 1973). If the radius at which a particle in a Keplerian orbit co-rotates with the star is smaller than the Alfvén radius, then centrifugal forces may not allow in-fall; rather, mass may be ejected and the outer magnetosphere may be unstable. In the other case, the accreting matter will fall toward the stellar surface in the vicinity of the magnetic poles, with a free fall time of about $0.1 \mathrm{~s}$. The pulsing of the X-ray emission is then due to the rotation of these hot spots on the neutron star. Although the basic spectrum of the pulses is presumably thermal with a temperature of about $5 \times 10^{7} \mathrm{~K}$, the details of both the spectrum and the pulse shape depend on, for example, the strength of the field, the density of the accretion column, and the possibilities of cyclotron radiation and Compton scattering (see e.g. Pringle and Rees, 1972; Davidson and Ostriker, 1973; Lamb et al., 1973; Gnedin and Sunyaev, 1973).

\subsection{ORIGIN AND EVOLUTION}

A final theoretical consideration is the fact that the origin and evolution of the components cannot be divorced from their presence in a binary system. At any time in the life span of one of the stars when expansion is expected, the presence of the other star will short-circuit the evolution when the expansion reaches the Roche lobe. At this point, significant mass transfer may start, changing the masses and subsequent evolution of both stars. Thus, stellar evolution in close binaries was of great interest even before the X-ray binaries were discovered (for a review, see e.g. Paczynski, 1971).

Specific evolutionary models for X-ray binaries have been produced (e.g. Van den Heuvel and Heise, 1972). One typically starts with a massive close binary system. The more massive component evolves faster and reaches its Roche lobe after some $10^{6}-10^{7} \mathrm{yr}$, before the onset of helium ignition. Very rapidly $\left(\sim 10^{4} \mathrm{yr}\right)$ most of the mass is transferred to the other component; the less massive component is now the more evolved star, and determines the final nature of the system. For small values of its mass, it becomes a degenerate dwarf. For larger values, it presumably undergoes a supernova explosion after another $\sim 10^{6} \mathrm{yr}$, forming a neutron star or black hole. The more massive star now continues its evolution, and if the binary system has 
survived the supernova, this star will reach its Roche lobe in $10^{6}-10^{7}$ yr and start transferring matter back onto the compact companion, generating $\mathrm{X}$-rays.

The crucial element in the evolution is the mass of the system. If the original mass of the system is low, one might expect to produce degenerate dwarfs or neutron stars with low mass companions, as has been discussed for Sco X-1, Cyg X-2, Cyg X-3, and Her X-1. Because of the low mass, the evolutionary times are very long, and the objects will be seen to belong to 'older' populations of stars as seems to be the case. If the original mass is high, one must end up with a high mass system as described above since the loss of more than half the original mass during a supernova explosion would disrupt a binary system. We do find high mass X-ray binary systems, as represented by Cyg X-1, Cen X-3, 3U 0900-40, 3U 1700-37, SMC X-1 and possibly Cir X-1. Because of the high mass, these systems are expected to evolve rapidly and to belong to a 'young' population as seems to be the case.

An important point of current investigation is the necessary condition for a supernova not to disrupt a binary system. A relevant consideration in this respect is the eccentricity of the X-ray binaries which would be expected as the result of a supernova explosion. Although there is some evidence for significant eccentricity in the optical light curve of, for example $3 \mathrm{U} 0900-40$, in the two X-ray pulsars Cen X-3 and Her $X-1$, upper limits for eccentricity on the order of $2 \times 10^{-3}$ have been obtained. Thus, further investigation is needed to determine how binaries can survive a supernova with very little or no eccentricity, or alternatively how any eccentricity can be efficiently removed.

Finally, we wish to point out the existence of a curious coincidence which has some bearing on the standard model. It is believed that neutron stars can only be produced explosively, i.e., during a supernova event. If this is correct, then wherever we see a neutron star in an X-ray source, there had to have been a supernova. In those instances where a black hole is in evidence, we would argue that the mass of the star undergoing the supernova was too massive to form a neutron star. The coincidence is simply that the known supernova show the same duplicity in population type as do the X-ray sources. The type II supernova occur only in spiral galaxies and are believed to originate in massive stars. Recent work by Moore (1973) has revealed that the supernova in spiral galaxies occur primarily at the edge of the spiral arms. The implication is that these supernovae have occurred within a few million years after the birth of the star which, thus, must be $\sim 20-40 \mathfrak{M}_{\odot}$. Moore's sample includes both type I, type II and unclassified supernova, but the evidence favours it being the type II events which occur at the arm edges. These objects could be precisely those we now see as the X-ray sources such as Cyg X-1 and Cen X-3 in association with O-B supergiants.

Regarding type I, it has been noted that they occur in all galaxy types and that their progenitors belong to a disk population or perhaps a mixed population (Tammann, 1974). This could be the same class of objects giving rise to X-ray sources found in association with low mass and population II objects, and also possibly a mixed population. Carrying this further, it has been noted for many years that the type $I$, 
because of their photometric uniformity, may represent a distinct transition. It has also been noted that a very attractive transition is a white dwarf at (or slightly above) its mass limit collapsing to a neutron star, as can happen in a binary system composed of a white dwarf which is accreting matter from its neighbor star. (See Schatzman, 1963; Whelan and Iben, 1973; Warner, 1974.)

Now what would be the result of such a transition taking place in a binary system? Obviously the X-ray source Her X-1 and its companion HZ Her are just what one might expect! It is also possible that the remaining low mass $\mathrm{X}$-ray sources arise by this means and their great variety simply reflects the variety of binary systems which can contain a white dwarf near its mass limit.

Like many arguments in astronomy, this one is somewhat circular and based on little direct evidences. However, considering that there are independent arguments for supernovae progenitors for X-ray sources, the occurrence of this coincidence is supporting evidence for $\mathrm{X}$-ray sources being neutron stars and black holes. In any event, the idea of a direct correspondence between binary $\mathrm{X}$-ray sources and supernovae events may be a fruitful working hypothesis for future investigations.

\section{Summary}

When the X-ray sources were first discovered there was the general belief that some very special conditions were required for their origin. It is now possible that we have discovered those conditions - supernova explosions, close binary systems, neutron stars and black holes. Where there is unambiguous data, this view is supported, in particular, by the existence of the pulsating $X$-ray sources and the mass estimate of Cyg X-1 and the direct evidence for the binary nature of many of the X-ray sources.

In addition, there are compelling theoretical arguments to support this view including the evolution of stars in close binary systems, the physics of neutron stars and black holes, and the great difficulty in finding alternative hypotheses to the above view.

However, one can not leave this subject without citing the problems, which are still formidable. There are details of the $\mathrm{X}$-ray and optical systems, which appear to be very definite, but as yet can not be simply explained. As an example, there is the wealth of information on Hercules X-1 - the 35-day cycle, the optical variability which are unexplained. The most puzzling feature is that the very large optical variability continues even when the $\mathrm{X}$-rays have disappeared. Is there yet another significant source of energy in the system, such as cosmic rays?

Other, less dramatic observations that require explanation are the two 'states' seen in many X-ray sources. In Cyg X-1, for example, one of the states is characterized by high intensity and a soft spectrum; in the other state the intensity is about a factor of three lower. Certain sources actually seem to 'disappear' from time to time. This has been seen in X-rays in Cir X-1 and SMC X-1. The most dramatic example of this is the case of Her $\mathrm{X}-1$, in which the optical variability disappears for years at a time, based on the study of historical material. 
This varied and complex behavior may be attributable to the complexity of the accretion process - instabilities in the atmosphere of the companion star and structure in the immediate vicinity of the X-ray source. Right now, however, these phenomena have no satisfactory explanation.

In spite of the difficulties in understanding the details of these objects, we can not overlook the potential of the field, principally the possibility of studying the properties of neutron stars and black holes at first hand, and of learning about a branch of the evolution of stars, following their supernova phase, that has completely eluded us up to now.

Although we have tried to acknowledge the most relevant references for the observations and their theoretical interpretations, we apologize for any significant omissions. In addition, we have made use of much as yet unpublished data from the Uhuru satellite, particularly in respect to Cen X-3, Cyg X-3, and Cir X-1. We thus acknowledge the efforts of the Uhuru group, and in particular, R. Giacconi, H. Tananbaum, S. Murray, W. Forman, C. Jones-Forman, R. Leach, G. Fabbiano, J. Morin, and R. Levinson. We would also like to thank D. Jarmac for assistance in preparing the manuscript.

\section{Appendix}

\section{Galactic $X$-ray sources}

A ( $I>8 \mathrm{ct} \mathrm{s}^{-1}$, non-identified with extragalactic objects)

$\begin{array}{llll}l, b & 3 \mathrm{U} & I_{\max } & \begin{array}{l}I_{\max } / \text { Comments } \\ I_{\min }\end{array}\end{array}$

Central Region of Galaxy

\begin{tabular}{|c|c|c|c|c|c|}
\hline $321,-6$ & $1543-62$ & 36 & 3 & & \\
\hline $391,-13$ & $1626-67$ & 10 & & & \\
\hline $322, \quad 0$ & $1516-56$ & 720 & $\geqslant 20$ & $\begin{array}{l}\text { Rapid fluctuations, } \\
\text { eclipse } 12.3 \text { days }\end{array}$ & Cir X-1 \\
\hline $324,-11$ & $1632-64$ & 11 & & & \\
\hline $324,-5$ & $1556-60$ & 17 & & SAO 253382 & B9, $8.9 \mathrm{~m}(?)$ \\
\hline $327+2$ & $1538-52$ & 11 & & & \\
\hline $332,-4$ & $1636-53$ & 261 & & & \\
\hline $335, \quad 0$ & $1624-49$ & 50 & 5 & $\begin{array}{l}\text { SAO } 226781 \\
\text { star cluster, NGC } 6134\end{array}$ & B8, $9 \mathrm{~m}(?)$ \\
\hline 336, & $1630-47$ & 150 & 3 & & \\
\hline $339,-4$ & $1658-48$ & 344 & 3 & & \\
\hline $340, \quad 0$ & $1642-45$ & 381 & 3 & & \\
\hline $343,-2$ & $1705-44$ & 280 & 3 & & \\
\hline $344,-1$ & $1702-42$ & 34 & & & \\
\hline $348,+2$ & $1700-37$ & 102 & $>3$ & $\begin{array}{l}\text { rapid fluctuations, } \\
\text { eclipse } 3.4 \text { days }\end{array}$ & $\begin{array}{l}\text { HD } 153919 \\
6 \mathrm{~m} \mathrm{O7f}\end{array}$ \\
\hline $346,-7$ & $1735-44$ & 210 & & & \\
\hline $348,-1$ & $1714-39$ & 12 & & & \\
\hline $349,+3$ & $1702-36$ & 715 & 2 & & \\
\hline $353,+5$ & $1704-32$ & 14 & & & \\
\hline $354,-5$ & $1746-37$ & 31 & & SAO 209318 & $\begin{array}{l}\mathrm{K} 1,3.2 \mathrm{~m} \\
\text { Glob. cluster NGC } 64\end{array}$ \\
\hline
\end{tabular}


A $\left(I>8 \mathrm{ct} \mathrm{s}^{-1}\right.$, non-identified) Continued

\begin{tabular}{llll}
\hline$l, b$ & $3 \mathrm{U}$ & $I_{\max }$ & $\begin{array}{c}I_{\max } / \text { Comments } \\
I_{\min }\end{array}$
\end{tabular}

Central Region of Galaxy

\begin{tabular}{rrrll}
354,0 & $1727-33$ & \multicolumn{1}{l}{65} & & \multicolumn{1}{l}{ SAO 208881 } \\
SAO 208883 \\
$357,-11$ & $1822-37$ & 17 & & \\
$357,-5$ & $1755-33$ & 47 & 3 & \\
$359,+24$ & $1617-15$ & 17,000 & 2.5 & \\
$0,+9$ & $1709-23$ & 39 & 5 & \\
$2,+5$ & $1728-24$ & 60 & & \\
$2,+1$ & $1744-26$ & 460 & 3 & \\
$3,-8$ & $1800-30$ & 250 & 1.5 & Glob. Cluster, NGC 6624? \\
$5,-1$ & $1758-25$ & 1127 & 2 & SAO 186106 \\
$8,+9$ & $1728-16$ & 260 & 1.7 & \\
$9,+1$ & $1758-20$ & 595 & 2 & \\
$14,+0$ & $1811-17$ & 380 & 3 & \\
$16,+1$ & $1813-14$ & 588 & 1.5 & \\
$18,+2$ & $1812-12$ & 12 & & \\
$30,+6$ & $1822-00$ & 37 & & \\
$31,-8$ & $1915-05$ & 23 & 5 & \\
$36,-4$ & $1908+00$ & 199 & 3 & \\
$36,+5$ & $1837+04$ & 270 & 2 & \\
$37,-1$ & $1901+07$ & 87 & 4 &
\end{tabular}

B5, $6.7 \mathrm{~m}$

B8, $9.4 \mathrm{~m}$ (?)

Sco X-1, 12.3 mag. pec.

$\mathrm{K} 7,8.8 \mathrm{~m}$ ?

Outer region of galaxy

\begin{tabular}{|c|c|c|c|c|}
\hline $43,-2$ & $1912+07$ & 21 & & \\
\hline $51,-9$ & $1956+11$ & 17 & & \\
\hline $68,+2$ & $1953+31$ & 63 & 5 & \\
\hline $58,+38$ & $1653+35$ & 100 & $\geqslant 6$ & $\begin{array}{l}1.2 \mathrm{~s} \text { pulses, } \\
\text { eclipses } 1.7 \text { days }\end{array}$ \\
\hline $71,+3$ & $1956+35$ & 1175 & 5 & $\begin{array}{l}\text { Cyg X-1 } \\
\text { Rapid fluctuations }\end{array}$ \\
\hline $80,+1$ & $2030+40$ & 194 & $\geqslant 3$ & $\begin{array}{l}\text { Cyg X-3, IR source } \\
\text { Partial eclipse (?) } 0.2 \text { days }\end{array}$ \\
\hline $87,-11$ & $2142+38$ & 540 & $\geqslant 2.5$ & Cyg X-2, $14 \mathrm{~m}$ sdG \\
\hline $92,-3$ & $2129+47$ & 12 & & \\
\hline $126,+1$ & $0115+63$ & 70 & 7 & \\
\hline $163,-17$ & $0352+30$ & 20 & & \\
\hline $201,-3$ & $0614+09$ & 60 & 6 & \\
\hline $263,+4$ & $0900-40$ & 100 & 10 & $\begin{array}{l}\text { HD } 77581,6 \mathrm{~m}, \mathrm{~B} 0.5 \mathrm{Ib} \\
\text { slow flares, } \\
\text { eclipses, } 8.9 \text { days }\end{array}$ \\
\hline $263,-11$ & $0750-49$ & 9 & & \\
\hline $283,+1$ & $1022-55$ & 10 & & \\
\hline $292, \quad 0$ & $1118-60$ & 160 & $\geqslant 20$ & $\begin{array}{l}\text { Cen } \mathrm{X}-3,6 \mathrm{~m}, \mathrm{~B} 0 \mathrm{Ib} \\
4.8 \text {-s pulses, } \\
\text { eclipses } 2.1 \text { days }\end{array}$ \\
\hline 294, & $1134-61$ & 9 & & \\
\hline 296, & $1145-61$ & 72 & 5 & $\begin{array}{l}\text { SAO } 251590 \\
\text { SAO } 251595\end{array}$ \\
\hline $\begin{array}{l}300, \quad 0 \\
303,-6\end{array}$ & $\begin{array}{l}1223-62 \\
1254-59\end{array}$ & $\begin{array}{l}32 \\
26\end{array}$ & 3 & SAO 251905 \\
\hline $304,+1$ & $1258-61$ & 47 & 5 & \\
\hline
\end{tabular}

Her X-1, Hz Her 13-15 mag. pec. HDE 226868

O9Ib, 9m
B0, $9.2 \mathrm{~m}$

B2, $8.6 \mathrm{~m}($ ?)

A1, $9.9 \mathrm{~m}($ (?) 
A $\left(I<8 \mathrm{ct} \mathrm{s}^{-1}, b< \pm 5^{\circ}\right.$, non-identified) Continued

$l, b \quad 3 \mathrm{U} \quad I_{\max } \quad I_{\max } /$ Comments

$I_{\min }$

Magellanic Cloud sources

\begin{tabular}{lrlll}
$0115-37$ & \multicolumn{1}{c}{28} & $\geqslant 9$ & SMC & $\begin{array}{l}\text { Sanduleak 160, } \\
13 \mathrm{~m}, \mathrm{~B} 01 \mathrm{~b}, \\
\text { eclipses 3.9 days }\end{array}$ \\
$0532-66$ & 9.4 & & LMC & \\
$0539-64$ & 20.7 & & LMC & \\
$0540-69$ & 19.3 & & LMC &
\end{tabular}

B $\left(I<8 \mathrm{ct} \mathrm{s}^{-1}, b< \pm 5^{\circ}\right.$, non-identified $)$

\begin{tabular}{lll}
$l, b \quad 3 \mathrm{U}$ & $I_{\max }$ & $\begin{array}{l}I_{\max } / \text { Comments } \\
I_{\min }\end{array}$ \\
\hline
\end{tabular}

$\begin{array}{rrrrr}320,-1 & 1510-59 & 6.4 & \text { SAO } 242322 & \text { G0, } 10 \mathrm{~m}(?) \\ 26,+1 & 1832-05 & 6.1 & & \\ 44,+1 & 1906+09 & 7.6 & & \\ 88,+2 & 2052+47 & 6.2 & \end{array}$

$101,-1 \quad 2208+54 \quad 4.4$

$107,+1 \quad 2233+59 \quad 4.7$

$130,-1 \quad 0143+61 \quad 7.2$

$\begin{array}{lll}138,+2 & 0258+60 & 2.9\end{array}$

$143,-4 \quad 0305+53 \quad 2.8$

$143,-1 \quad 0318+55 \quad 4.9$

$\begin{array}{llll}161, & 0 & 0446+44 & 6.2\end{array}$

$189,+5 \quad 0620+23 \quad 5.0$

$244,+2 \quad 0757-26 \quad 3.0$

$276,-4 \quad 0918-55 \quad 6.3$

SAO $236863 \quad$ A0, $10 \mathrm{~m}$

$294, \quad 0 \quad 1134-61 \quad 8.7$

SAO $236859 \quad$ K0, $8.1 \mathrm{~m}($ ?)

$299,-2 \quad 1210-64 \quad 6.0$

C (Other galactic sources)

Name $3 \mathrm{U}$ designation $I(2-6 \mathrm{keV}) \quad$ Comments

Supernova Remnants

$\begin{array}{llcl}\text { Crab Nebula } & 0531+21 & 950 & \\ \text { NP 0532 } & - & - & \\ \text { Cas A } & 2321-58 & 53 & \\ \text { Tycho } & 0022+63 & 9.5 & \\ \text { Puppis A } & 0821-42 & 7.5 & \text { Very soft spectrum } \\ \text { Vela X, Y, Z } & 0833-45 & 9.1 & \\ \text { Cygnus Loop } & - & - & \\ \text { Lupus SN? } & - & - & \\ \text { IC 443? } & 0620+23 & 6.0 & \\ \text { MSH 15-52 A? } & 1510-59 & 6.4\end{array}$

Galactic Center

GCX $\quad 1743-29 \quad 40 \quad$ Extended $\sim 1^{\circ}$


C (Other galactic sources) Continued

\begin{tabular}{llll}
\hline Name & 3U designation & Duration & $I$ (peak) \\
\hline Transient Sources & & & \\
Cen X-2 & & $\sim 80$ days & $5 \times 10^{-7} \mathrm{erg} \mathrm{cm}^{-2} \mathrm{~s}^{-1}$ \\
Cen X-4 & & $\sim 80$ days & $5 \times 10^{-7}$ \\
& $1543-47$ & $\sim 1$ yr & $3 \times 10^{-8}$ \\
Cep X-4 & $1735-28$ & $\sim 1$ week & $10^{-8}$ \\
\hline
\end{tabular}

\section{References}

Arons, J.: 1973, Astrophys. J. 184, 539.

Bahcall, J. and Bahcall, N.: 1972, Astrophys. J. 178, L1.

Baity, W., Ulmer, M., Wheaton, W., and Peterson, L.: 1974, Astrophys. J. 187, 341.

Basko, M. and Sunyaev, R.: 1973, Astrophys. Space Sci. 23, 117.

Basko, M., Sunyaev, R., and Titarchuk, L.: 1974, Astron. Astrophys. 31, 249.

Becklin, E. and Neugebauer, G.: 1974, private communication.

Becklin, E., Hawkins, F. J., Mason, K. O., Matthews, K., Neugebauer, G., Sanford, P. W., and Wynn-Williams, C.: 1973, Nature 245, 302.

Blaauw, A.: 1965, in A. Blaauw and M. Schmidt (eds.), 'Galactic Structure', Stars and Stellar Systems 5, 435 (Univ. of Chicago Press).

Blaauw, A.: 1961, Bull. Astron. Inst. Neth. 15, 265.

Blumenthal, G. and Tucker, W.: 1972, Nature 235, 97.

Boldt, E., Holt, S., and Serlemitsos, P.: 1971, Astrophys. J. 164, L9.

Bolton, C.: 1972, Nature 235, 271.

Boynton, P. C., Canterna, R., Crosa, L., Deeter, J., and Gerend, D.: 1973, Astrophys. J. 186, 617.

Bowyer, S., Byram, T., Chubb, T., and Friedman, H.: 1964, Science 146, 912.

Braes, L. and Miley, G.: 1971, Nature 232, 246.

Braes, L. and Miley, G.: 1972, Nature 237, 506.

Braes, L., Miley, G., Shane, W., Baars, J., and Goss, M.: 1973, Nature Phys. Sci. 242, 66.

Bregman, J., Butler, D., Kemper, E., Koski, A., Kraft, R., and Stone, R.: 1973, Astrophys. J. $185, \mathrm{~L} 117$.

Brinkman, A., Parsignault, D., Schreier, E., Gursky, H., Kellogg, E., Tananbaum, H., and Giacconi, R.: 1974, Astrophys. J. 188, 603.

Byram, E., Chubb, T., and Friedman, H.: 1966, Science 132, 66.

Cameron, A. and Maeder, M.: 1967, Nature 215, 464.

Canizares, C. R., McClintock, J. E., Clark, C. W., Lewin, W. H. G., Schnopper, H. W., and Sprott, G. F.: 1973, Nature Phys. Sci. 241, 28.

Cherepashchuk, A. M., Luyty, V. M., and Sunyaev, R. A.: 1973, Astron. Zh. $50,3$.

Chevalier, C. and Ilovaisky, S.: 1974, Astron. Astrophys. 35, 407.

Clark, G. W., Bradt, H. V., Lewin, W. H. G., Markert, T. H., Schnopper, H. W., and Sprott, G. F.: 1972, Astrophys. J. 177, L109.

Crampton, D. and Hutchings, J. B.: 1972, Astrophys. J. 178, L65.

Davidsen, A., Henry, J., Middleditch, J., and Smith, H.: 1972, Astrophys. J. 177, L97.

Davidson, K. and Ostriker, J.: 1973, Astrophys. J. 179, 585.

Davidson, K. and Ostriker, J.: 1974, Astrophys. J. 189, 431.

Dilworth, C., Maraschi, L., and Reina, C.: 1974, Astron. Astrophys. 28, 71.

Doxsey, R., Bradt, H. V., Levine, A., Murthy, G. T., Rappaport, S., and Spada, G.: 1973, Astrophys. J. 182, L25.

Fabian, A., Pringle, J., and Rees, M.: 1973, IAU Circ., No. 2555.

Felten, J. E., Rees, M. J., and Adams, T. F.: 1972, Astron. Astrophys. 21, 139. 
Fisher, P. C., Johnson, H. M., Jordan, W. C., Meyerott, A. J., and Acton, L. W.: 1966, Astrophys. J. 148, L119.

Forman, W.: 1974, preprint.

Forman, W., Jones, C., and Liller, W.: 1972, Astrophys. J. 177, L103.

Forman, W., Jones, C., Tananbaum, H., Gursky, H., Kellogg, E., and Giacconi, R.: 1973, Astrophys. J. $182, \mathrm{~L} 103$.

Friedman, H., Byram, E., and Chubb, T.: 1967, Science 156, 374.

Giacconi, R., Gursky, H., Paolini, F. R., and Rossi, B. B.: 1962, Phys. Rev. Letters 9, 439.

Giacconi, R., Gorenstein, P., Gursky, H., Usher, P. D., Waters, J. R., Sandage, A., Osmer, P., and Peach, J.: 1967, Astrophys. J. 148, L119.

Giacconi, R., Gursky, H., Kellogg, E., Schreier, E., and Tananbaum, H. : 1971, Astrophys. J. 167 , L67.

Giacconi, R., Gursky, H., Kellogg, E., Levinson, R., Schreier, E., and Tananbaum, H.: 1973, Astrophys. J. 184, 227.

Giacconi, R., Murray, S., Gursky, H., Kellogg, E., Schreier, E., Matilsky, T., Koch, D., and Tananbaum, H.: 1974, Astrophys. J. (Suppl.) 237, 27, 37.

Gnedin, Y. and Sunyaev, R.: 1973, Astron. Astrophys. 25, 233.

Gregory, P., Kronberg, P., Seaquist, E., Hughes, V., Woodsworth, A., Viner, M., and Retallack, D. : 1972, Nature 239, 440.

Groth, E., Yeung, S., Papaliolios, C., Pennypacker, R. C., Spada, G., and Middleditch, J.: 1973, IAU Circ., No. 2523.

Gursky, H.: 1972, Astrophys. J. 175, L141.

Gursky, H.: 1973, in C. DeWitt and B. S. DeWitt (eds.), Black Holes, Gordon and Breach, New York.

Gursky, H., Gorenstein, P., and Giacconi, R.: 1967, Astrophys. J. 150, L75.

Hayakawa, S. and Matsuoka, M.: Prog. Theoret. Phys. 1964, Suppl. Japan, No. 30, 204.

Hensberge, G., van den Heuvel, E. P. J., and Paes de Barros, M. H.: 1973, Astron. Astrophys. $29,69$.

Hiltner, W., Werner, J., and Osmer, P.: 1972, Astrophys. J. 175, L19.

Hjellming, R. M.: 1973, Astrophys. J. 182, L29.

Hjellming, R. M. and Balick, B.: 1972, Nature 239, 443.

Hjellming, R. M. and Wade, C.: 1971, Astrophys. J. 168, L21.

Holt, S., Boldt, E., Schwartz, D., Serlemitsos, P., and Bleach, R.: 1971, Astrophys. J. 166, L65.

Holt, S., Boldt, E., Rothschild, R., and Serlemitsos, P.: 1974, preprint.

Hutchings, J. P.: 1972, IAU Circ., No. 2537.

Hutchings, J. B., Thackeray, A. D., Webster, B. L., and Andrews, P. T.: 1973, Monthly Notices Roy. Astron. Soc. 163, 13.

Jones, C.: 1974, preprint.

Jones, C. and Liller, W. : 1973, Astrophys. J. 184, L65.

Jones, C., Forman, W., Tananbaum, H., Schreier, E., Gursky, H., Kellogg, E., and Giacconi, R.: 1973a, Astrophys. J. 181, L43.

Jones, C., Forman, W., and Liller, W.: 1973b, Astrophys. J. 182, L109.

Jones, C., Giacconi, R., Forman, W., and Tananbaum, H.: 1974, Astrophys. J. 191, L71.

Joss, P. C., Avni, Y., and Bahcall, J.: 1973, Astrophys. J. 186, 767.

Kopal, Z.: 1959, Close Binary Systems, Wiley, New York.

Kraft, R. and Demoulin, M.: 1967, Astrophys. J. 150, L183.

Kruszewski, A.: 1966, Adv. Astron. Astrophys. 4, 233 (Academic Press, New York).

Krzeminsky, W.: 1973, IAU Circ. No. 2569.

Lamb, D. and Sorvari, J. M.: 1972, IAU Circ., No. 2422.

Lamb, F. K., Pethick, C., and Pines, D.: 1973, Astrophys. J. 184, 271.

Li, F. K. and Clark, G. W.: 1974, preprint.

Liller, W.: 1972a, IAU Circ., No. 2415.

Liller, W.: 1972b, IAU Circ., No. 2427.

Liller, W.: 1972c, 'Tabulation of Interesting Optical and Radio Sources near Uhuru X-ray Sources', Harvard College Observatory.

Liller, W.: 1973, Astrophys. J. 184, L37.

Margon, B. and Ostriker, J.: 1973, Astrophys. J. 186, 91.

Margon, B., Lampton, M., Bowyer, S., and Cruddace, R.: 1971, Astrophys. J. 169, L23.

Margon, B., Bowyer, S., and Stone, R.: 1973, Astrophys. J. 185, L113. 
Matteson, J.: 1971, Ph.D. Thesis, U.C., San Diego.

Mauder, H.: 1973, Astron. Astrophys. 28, 473.

Middleditch, J. and Nelson, J.: 1973, preprint.

Miyamoto, S., Fujii, M., Matsuoka, M., Nishimuru, J., Oda, M., Ogawara, Y., Ohta, S., and Wada, M.: 1971, Astrophys. J. 168, L11.

Moore, E.: 1973, Publ. Astron. Soc. Pacific 85, 564.

Neugebauer, G., Oke, J., Becklin, E., and Garmire, G.: 1969, Astrophys. J. 155, 1.

Oda, M. and Matsuoka, M.: 1970, Prog. Elem. Part. Cosmic-Ray Phys. 10, 305.

Oda, M., Gorenstein, P., Gursky, H., Kellogg, E., Schreier, E., Tananbaum, H., and Giacconi, R.: 1971, Astrophys. J. 166, L1.

Oda, M., Wada, M., Matsuoka, M., Miyamoto, S., Muranaka, N., and Ogawara, Y.: 1972, Astrophys. J. 172, L13.

Osmer, P. and Hiltner, W.: 1974 Astrophys. J. 188, L5.

Ostriker, J.: 1971, Ann. Rev. Astron. Astrophys. 9, 353.

Overbeck, J. and Tananbaum, H.: 1968, Phys. Rev. Letters 20, 24.

Paczynski, B.: 1971, Ann. Rev. Astron. Astrophys. 9, 183.

Paczynski, B.: 1973, Ann. N.Y. Acad. Sci. 224, 233.

Parsignault, D. R., Gursky, H., Kellogg, E., Matilsky, T., Murray, S., Schreier, E., Tananbaum, H., Giacconi, R., and Brinkman, A. C.: 1972, Nature Phys. Sci. 239, 123.

Peterson, L.: 1973, in H. Bradt and R. Giacconi (eds.), 'X- en Gamma-Ray Astronomy' IAU Symp. 55, 51.

Prendergast, K. and Burbidge, G.: 1968, Astrophys. J. 151, L83.

Price, R. E., Groves, D. S., Rodrigues, R. M., Seward, F., Swift, C. D., and Toor, A.: 1971, Astrophys. J. 168, L7.

Pringle, J.: 1973, Nature Phys. Sci. 243, 90.

Pringle, J. and Rees, M.: 1972, Astron. Astrophys. 21, 1.

Rappaport, S., Doxsey, R., and Zaumen, W.: 1971a, Astrophys. J. 168, L43.

Rappaport, S., Zaumen, W., and Doxsey, R.: 1971b, Astrophys. J. 168, L17.

Rothschild, R., Boldt, E., Holt, S., and Serlemitsos, P.: 1974, preprint.

Ruffini, R.: 1973, in C. DeWitt and B. S. DeWitt (eds.), Black Holes, Gordon and Breach, New York. Ryter, C.: 1970, Astron. Astrophys. 9, 288.

Salpeter, E.: 1973, in H. Bradt and R. Giacconi (eds.), 'X- and Gamma Ray Astronomy', IAU Symp. 55, 135.

Sanford, P.: 1973, preprint.

Sanford, P., Mason, K., Hawkins, F., Murdin, P., and Savage, A.: 1974, preprint.

Schatzman, E.: 1963, in L. Gratter (ed.), Star Evolution, Proc. Int. School of Phys. 'Enrico Fermi', Academic Press, New York, p. 389.

Schreier, E., Gursky, H., Kellogg, E., Tananbaum, H., and Giacconi, R.: 1971, Astrophys. J. 170, L21.

Schreier, E., Levinson, R., Gursky, H., Kellogg, E., Tananbaum, H., and Giacconi, R.: 1972a, Astrophys. J. 172, L79.

Schreier, E., Giacconi, R., Gursky, H., Kellogg, E., and Tananbaum, H.: 1972b, Astrophys. J. 178, L71.

Setti, G. and Woltjer, L.: 1970, Astrophys. Space Sci. 9, 185.

Seward, F. D., Burginyon, G. A., Grader, R., Hill, R., and Palmieri, T.: 1972, Astrophys. J. 178, 131.

Shakura, N. I. and Sunyaev, R. A.: 1973, Astron. Astrophys. 24, 337.

Shklovsky, I.: 1967, Astrophys. J. 148, L1.

Shulman, S., Fritz, G., Meekins, J., and Friedman, H.: 1971, Astrophys. J. 168, L49.

Shvartsman, V. F.: 1971, Soviet Astron. - A.J. 15, 342. = Astron. Zh. 48, 438.

Stockman, S. H., Angel, J. R. P., Novick, R., and Woodgate, B. E.: 1973, Astrophys. J. 183, L63.

Strittmatter, P., Scott, J., Whelan, J., Wickramasinghe, D., and Woolf, N.: 1973, Astron. Astrophys. 25, 275.

Sunyaev, R.: 1973, Soviet Astron. - A.J. (in press).

Tammann, G. A.: 1974, in C. B. Cosmovici (ed.), Supernovae and Supernova Remnants, D. Reidel, Dordrecht, Holland, p. 155.

Tananbaum, H.: 1974, in F. J. Kerr and S. C. Simonson III (eds.), 'Galactic Radio Astronomy', IAU Symp. 60, 383. 
Tananbaum, H., Gursky, H., Kellogg, E., Giacconi, R., and Jones, C.: 1972a, Astrophys. J. 177, 15. Tananbaum, H., Gursky, H., Kellogg, E., Levinson, R., Schreier, E., and Giacconi, R.: 1972b, Astrophys. J. 174, L143.

Trimble, V., Rose, W., and Weber, W.: 1973, Monthly Notices Roy. Astron. Soc. $162,1$.

Ulmer, M., Baity, W., Wheaton, W., and Peterson, L.: 1972a, Astrophys. J. 178, L61.

Ulmer, M., Baity, W., Wheaton, W., and Peterson, L.: 1972b, Astrophys. J. 178, L121.

Van den Heuvel, E. and Heise, J.: 1972, Nature Phys. Sci. 239, 67.

Van den Heuvel, E. and Ostriker, J.: 1973, Nature Phys. Sci. 245, 99.

Wade, C. and Hjellming, R.: 1971, Astrophys. J. 170, 523.

Warner, B.: 1974, Monthly Notices Roy. Astron. Soc. 167, 61 pp.

Webster, B. and Murdin, P.: 1972, Nature 235, 37.

Webster, B., Martin, W., Feast, M., and Andrews, P.: 1972, Nature Phys. Sci. 240, 183.

Whelan, J. and Iben, I.: 1973, Astrophys. J. 186, 1007.

Wilson, R.: 1970, in L. Gratton (ed.), 'Non-Solar X- and Gamma-Ray Astronomy', IAU Symp. 37, 242.

Wilson, R.: 1973, Astrophys. J. 181, L75.

Wolf, S. C. and Morrison, N. D.: 1973, Publ. Astron. Soc. Pacific, 85, 141.

\section{DISCUSSION}

$P$. $R$. Amnuel: What can you say about the possibility of the existence of a faint X-ray sources class in the Galaxy?

$H$. Gursky: The brightest source SMC X-1 has a luminosity of $\sim 10^{39} \mathrm{erg} \mathrm{s}^{-1}$. The majority of identified sources have $L \approx 10^{37}-10^{38} \mathrm{erg} \mathrm{s}^{-1}$. But the instrument makes it possible to observe considerably fainter galactic sources and they are really observed.

W. Seggewiss: You indicate that there are stars with normal abundance in the type II process but H-deficiency in type I. On the other hand, Wolf-Rayet stars (which are known to be H-deficient) may be involved in the type II process, if the evolutionary scheme of Van den Heuvel leading to $\mathrm{X}$-ray sources is correct.

H. Gursky: As I stated, this view of the relation between $\mathrm{SN}$ and $\mathrm{X}$-ray sources is very speculative. The question is a very serious one which must be answered by the experts in this field.

$E$. R. Mustel: Recently several papers were published on type I supernova models in which it is suggested that before their explosion these stars are composed of a normal star and a neutron star. What is your opinion on this?

H. Gursky: I cannot judge very well the evolutionary tracks which were derived in these cases. I think that the important point is the observational evidence, namely the existence of stellar systems combining a white dwarf and a normal or giant star and for mass transfer between the two; and the existence of very similar X-ray emitting stellar systems, except for the substitution of a neutron star or black hole instead of a white dwarf. Finally the mass limit of white dwarfs is well established both theoretically and observationally. Thus we see all the elements required to make type I SN, the initial conditions, the final conditions and the intervening physics.

B. $V$. Komberg: It's a bit strange that Her X-1 and Cen X-3 X-ray pulsars have similar X-ray properties; at the same time, they are put into different classes in your table. How would you explain this?

$H$. Gursky: I don't know why it is so. The physics of the radiation is similar, and the astronomy is different.

D. Y. Martynov: What's your opinion about the relation between X-ray stars (which sometimes are $\mathrm{X}$-ray pulsars) and radio pulsars?

Are they totally different objects or is there any evolutionary link between them? So far we've only one known linking object, the pulsar in the Crab Nebula.

H. Gursky: There is no apparent physical connection between the phenomena associated with radio pulsars and those seen in the pulsing X-ray sources such as Her X-1; both are likely to be rotating neutron stars possessing large magnetic fields; but the physical mechanism of radiation processes is probably very different. Regarding the evolution of these objects, it is very possible that the pulsars and the X-ray sources have common parents; in the case of the X-ray sources the binary system remains intact following a supernova explosion; in the case of the pulsar, the binary is disrupted following the supernova explosion. 
$Y u$. N. Gnedin: What can you say on the beaming of the X-ray radiation from the experimental data?

H. Gursky: The width of the beam can be $30^{\circ}-40^{\circ}$.

$O$. H. Gusinov: (1) The results of our works almost absolutely exclude the formation of neutron and collapsed stars without a supernova explosion. The formation of a neutron star is mainly the result of an SN explosion.

(2) In order to explain binaries where one component is a relatively small star and the second one is a massive star which can be observed in the optical, it's not necessary at all to say that there is the process of matter exchange and that as a result the less massive component becomes more massive. We can consider the matter to be lost from the system instead of being accreted onto the second component.

A star of any mass can be a companion of a degenerate star.

(3) There are $\sim 10^{4} \mathrm{X}$-ray sources with less than $10^{35} \mathrm{erg} \mathrm{s}^{-1}$ luminosity in the Galaxy. The usual components in these sources are stars of low mass. Some of these sources, $\sim 10-15$, are included in the Uhuru catalogue. 